\title{
Corotating solar wind streams and recurrent geomagnetic activity:
}

\section{A review}

\author{
Bruce T. Tsurutani, ${ }^{1,2}$ Walter D. Gonzalez, ${ }^{3}$ Alicia L. C. Gonzalez, ${ }^{3}$ \\ Fernando L. Guarnieri, ${ }^{3}$ Nat Gopalswamy, ${ }^{4}$ Manuel Grande, ${ }^{5}$ Yohsuke Kamide, ${ }^{6}$ \\ Yoshiya Kasahara, ${ }^{7}$ Gang Lu, ${ }^{8}$ Ian Mann, ${ }^{9}$ Robert McPherron, ${ }^{10}$ Finn Soraas, ${ }^{11}$ \\ and Vytenis Vasyliunas ${ }^{12}$
}

Received 16 June 2005; revised 3 October 2005; accepted 16 December 2005; published 29 June 2006.

[1] Solar wind fast streams emanating from solar coronal holes cause recurrent, moderate intensity geomagnetic activity at Earth. Intense magnetic field regions called Corotating Interaction Regions or CIRs are created by the interaction of fast streams with upstream slow streams. Because of the highly oscillatory nature of the GSM magnetic field $\mathrm{z}$ component within CIRs, the resultant magnetic storms are typically only weak to moderate in intensity. CIR-generated magnetic storm main phases of intensity Dst $<$ $-100 \mathrm{nT}$ (major storms) are rare. The elongated storm "recovery" phases which are characterized by continuous AE activity that can last for up to 27 days (a solar rotation) are caused by nonlinear Alfven waves within the high streams proper. Magnetic reconnection associated with the southward (GSM) components of the Alfvén waves is the solar wind energy transfer mechanism. The acceleration of relativistic electrons occurs during these magnetic storm "recovery" phases. The magnetic reconnection associated with the Alfvén waves cause continuous, shallow injections of plasma sheet plasma into the magnetosphere. The asymmetric plasma is unstable to wave (chorus and other modes) growth, a feature central to many theories of electron acceleration. It is noted that the continuous AE activity is not a series of substorm expansion phases. Arguments are also presented why these AE activity intervals are not convection bays. The auroras during these continuous AE activity intervals are less intense than substorm auroras and are global (both dayside and nightside) in nature. Owing to the continuous nature of this activity, it is possible that there is greater average energy input into the magnetosphere/ ionosphere system during far declining phases of the solar cycle compared with those during solar maximum. The discontinuities and magnetic decreases (MDs) associated with interplanetary Alfven waves may be important for geomagnetic activity. In conclusion, it will be shown that geomagnetic storms associated with high-speed streams/CIRs will have the same initial, main, and "recovery" phases as those associated with ICME-related magnetic storms but that the interplanetary causes are considerably different.

Citation: Tsurutani, B. T., et al. (2006), Corotating solar wind streams and recurrent geomagnetic activity: A review, J. Geophys. Res., 111, A07S01, doi:10.1029/2005JA011273.

\section{Introduction}

[2] During the declining phase of the solar cycle (see discussion of solar cycle phases in the glossary in

\footnotetext{
${ }^{1}$ Jet Propulsion Laboratory, Pasadena, California, USA.

${ }^{2}$ Also at Kyoto University, Uji, Japan.

${ }^{3}$ Instituto Nacional Pesquisas Espaciais (INPE), Sao Jose dos Campos, Sao Paolo, Brazil.

${ }^{4}$ NASA Goddard Space Flight Center, Greenbelt, Maryland, USA.

${ }^{5}$ Planets and Magnetospheres Group, Rutherford Appleton Laboratory, Oxfordshire, UK.

${ }^{6}$ Solar-Terrestrial Environment Laboratory, Nagoya University, Toyokawa, Japan.

Copyright 2006 by the American Geophysical Union. 0148-0227/06/2005JA011273
}

Appendix A) well away from solar maximum, the dominant solar phenomenon affecting geomagnetic activity at Earth is coronal holes. High-speed ( $\sim 50$ to $800 \mathrm{~km} / \mathrm{s}$ ) solar winds emanate continuously from these coronal holes [Krieger et

\footnotetext{
${ }^{7}$ Department of Information and Systems Engineering, Kanazawa University, Kanazawa, Japan.

${ }^{8}$ High Altitude Observatory, National Center for Atmospheric Research, Boulder, Colorado, USA.

${ }^{9}$ Department of Physics, University of Alberta, Edmonton, Alberta, Canada.

${ }^{10}$ Institute of Geophysics and Planetary Physics, University of California at Los Angeles, Los Angeles, California, USA.

${ }^{11}$ Department of Physics and Technology, University of Bergen, Bergen, Norway.

${ }^{12}$ Max Planck Institute for Solar System Research, Lindau, Germany.
} 
al., 1973; Neupert and Pizzo, 1974; McComas et al., 2002]. If the holes are long-lasting (more than 27 days), the highspeed streams will reappear each solar rotation, thus giving an impression that the streams are "corotating" with the Sun. If the high-speed streams overtake slower-speed $(\sim 300$ to $400 \mathrm{~km} / \mathrm{s}$ ) streams, as happens near the ecliptic plane, the high-speed stream-slow-speed stream interactions result in both magnetic field and plasma compressions at their interfaces [Pizzo, 1985; Balogh et al., 1999]. For magnetic storm activity, the most important interplanetary features are these intense magnetic field regions, called "corotating interaction regions," or CIRs [Smith and Wolfe, 1976; see also Balogh et al., 1999].

[3] When CIRs impinge upon the Earth's magnetosphere, they can cause magnetic storms, albeit only weak to moderate in intensity [Tsurutani et al., 1995]. At $1 \mathrm{AU}$, CIRs are usually formed adjacent to the heliospheric current sheet. The high plasma densities near the heliospheric current sheet or the heliospheric current sheet plasma sheet (HCSPS) [Winterhalter et al., 1994a] cause increases in ram pressure onto the magnetosphere. These can be detected as magnetic field $\mathrm{H}$ (horizontal) component increases by near-equatorial ground-based magnetometers, or storm "initial phases" prior to the storm main phases [Tsurutani and Gonzalez, 1997]. Since density increases of the HCSPS are gradual (also at $1 \mathrm{AU}$ there are typically no shocks at the leading edges of CIRs), the storm initial phases begin gradually without sudden impulses (SIs). The high-speed solar wind contains large-amplitude (nonlinear) $\Delta \mathbf{b} /|\mathbf{B}|=1$ to 2 Alfvén waves [Belcher and Davis, 1971; Tsurutani et al., 1994; Balogh et al., 1995]. Negative interplanetary magnetic field $B_{z}$ components of the Alfvén waves within high-speed streams lead to continuous auroral zone activity called "High-Intensity Long-Duration Continuous AE Activity" or HILDCAAs that can last for a few to 27 days [Tsurutani and Gonzalez, 1987] (however, it should be noted that most but not all high-speed streams have Alfvén waves with negative interplanetary magnetic field $\mathrm{Bz}$ components). The energy input into the magnetosphere-ionosphere system, averaged over a year, can be higher than during solar maximum years when interplanetary coronal mass ejections (ICMEs) dominate solar (and geomagnetic) activity [Sheeley et al., 1976, 1977; Tsurutani et al., 1995]. One of the most important space weather phenomena, the acceleration of relativistic electrons [Paulikas and Blake, 1979; Baker et al., 1986], occurs during high-speed streams/HILDCAAs. However, the exact mechanism for energization is in debate at this time. Observations and specific possible mechanisms and discussions thereof can be found in the work of Hudson et al. [1999], Li and Temerin [2001], Horne [2002], O'Brien et al. [2001], Meredith et al. [2003], Elkington et al. [2003], Trakhtengerts et al. [2003], and Summers et al. [2004].

[4] The purpose of this review is to present a summary of what is presently known concerning corotating solar wind streams and recurrent geomagnetic activity in the declining phase of the solar cycle. This complex solar-interplanetarymagnetospheric-ionospheric coupling topic, dealing with related plasma pheonomena from the Sun to the Earth, was the main focus of the Chapman Conference held in
Manaus, Amazonas, Brazil, 6 to 12 February 2005. The papers that follow this one (in this special issue) give further details on this very important space weather topic.

\section{Results}

\subsection{Coronal Holes, High-Speed Streams, and Alfvén Waves}

[5] Figure 1 is a YOHKOH image of the Sun in soft $\mathrm{X}$ rays. The dark regions of the surface have been called "coronal holes" because of the absence of hot electrons in these regions. Coronal holes are the origins of high-speed solar wind streams [Krieger et al., 1973]. The mechanism(s) for plasma heating, leading to these fast streams, is currently under intensive study. Other than the possibility of nanoflares [Parker, 1988], almost all other theories involve a scenario where solar plasma waves propagate up into the corona and then deposit their energy at these higher altitudes via wave damping (see a review by Hollweg and Isenberg [2002] for a discussion of resonant wave interactions). Nanoflares and other processes may be the ultimate source for these waves.

[6] Figure 2 is a plot of measured solar wind speeds as a function of heliolatitude [Phillips et al., 1995]. The measurements were taken from the SWICS instrument on board the Ulysses spacecraft. In the figure, the speed is indicated by the radial distance measured from the center of the Sun. The SOHO EIT solar disc image, the HAO Mauna Loa coronagraph image, and the SOHO LASCO coronagraph image (for one point in time) are also included for visual context. All data were taken during the declining phase of the solar cycle. The solar wind speeds are for the years 1993 to 1995, i.e., through the first southern and first northern Ulysses polar passes. The important feature to note in the figure is that over both solar poles, there are large coronal holes (dark photospheric regions). The solar wind coming from these regions has a nearly constant speed of $\sim 750-800 \mathrm{~km} / \mathrm{s}$. At lower, $<30^{\circ}$ heliographic latitudes, the solar corona is dominated by helmet streamers. The slow-speed $(\sim 300-400 \mathrm{~km} / \mathrm{s})$, high plasma density solar wind comes from these general regions. The slow solar wind source is either the helmet steamers or their boundaries or both (see Suess and Nerney [2002] and Nerney and Suess [2005] for references to various specific mechanisms).

[7] The magnetic field polarity is indicated by the color of the solar wind speed data. Outward-from-the-Sun magnetic field polarities are detected in the northern hemisphere, and inward fields are detected in the southern hemisphere (these solar hemispherical polarities reverse every $\sim 11$ years or half the $\sim 22$ year solar cycle). At this time (declining phase of the solar cycle), the change of magnetic field polarity occurs near the solar equatorial region. The interplanetary manifestation of this magnetic field directional reversal is called the heliospheric current sheet, or HCS.

[8] The fast solar wind and its microscale structure are displayed in greater detail in Figure 3. Thirty days of plasma velocity and magnetic fields are given for the first south polar pass of Ulysses. The velocity and magnetic field components are in solar heliospheric coordinates, where $\mathbf{r}$ is the radial direction from the Sun to the spacecraft, $\mathbf{t}=$ 


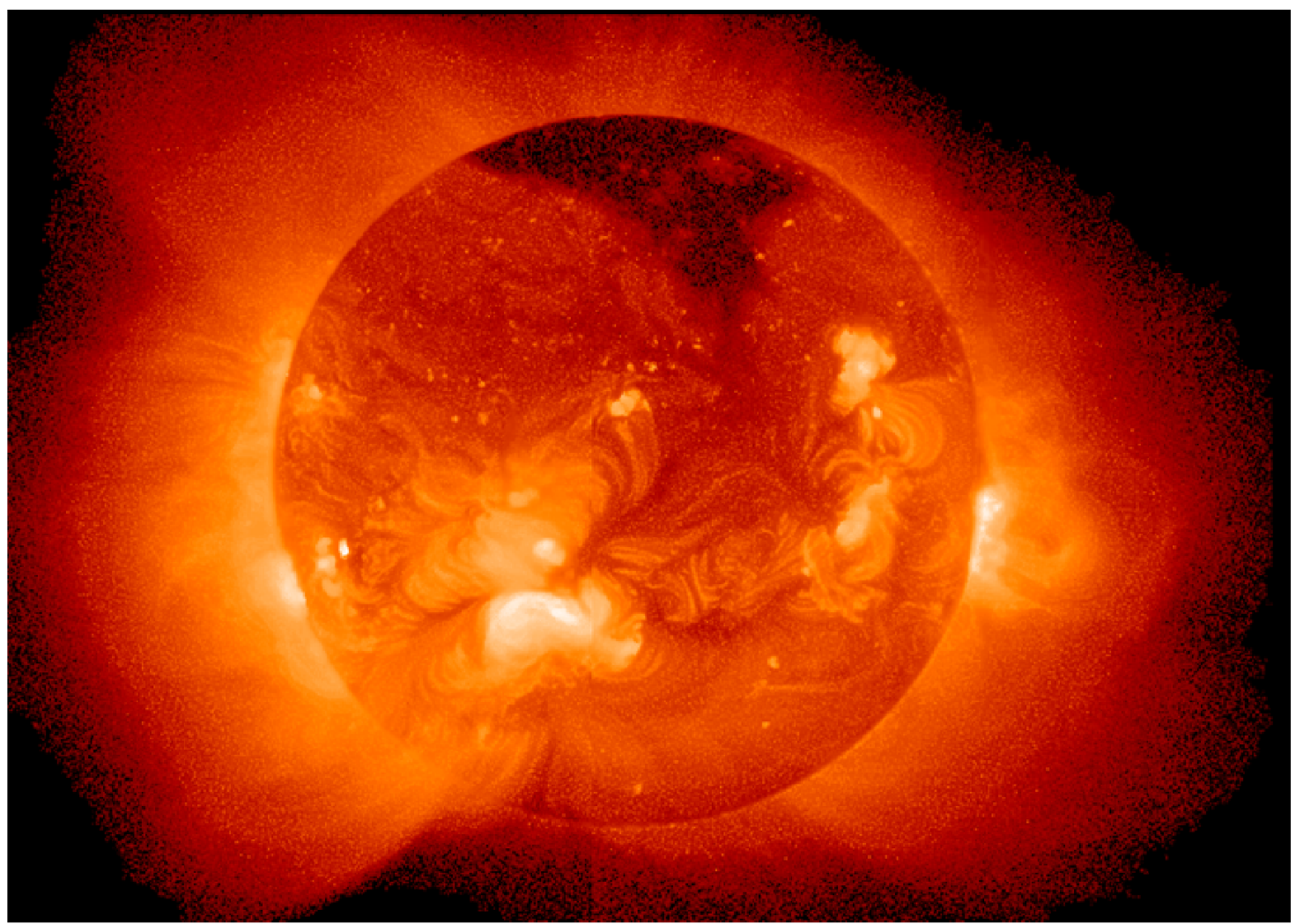

Figure 1. A Yohkoh image of the Sun taken in soft X rays. The dark regions are coronal holes. These are the sources of the high-speed solar wind streams.

$(\hat{\Omega} \times \mathbf{r}) /|\hat{\Omega} \times \mathbf{r}|$, where $\hat{\Omega}$ is the north rotation pole of the Sun, and $\hat{n}$ completes a right-hand coordinate system. The seventh panel from the top shows the nearly constant, high-speed solar wind emanating from the polar region of the Sun. The bottom panel gives the spacecraft heliographic latitude. Ulysses reached a minimum negative latitude of $\sim-80^{\circ}$.

[9] The large fluctuations in the magnetic field and solar wind velocity components (top six panels) are large-amplitude, nonlinear Alfvén waves (see discussion in the work of Belcher and Davis [1971] and Tsurutani et al. [2005]). These waves are propagating outward from the Sun [Balogh et al., 1995], but because the solar wind convection speed is much larger than the Alfvén wave phase speed (this is correct for linear waves; however, for nonlinear waves the Alfvén wave speed could be substantially larger), the wave are largely convected radially outward from the Sun. The peak-to-peak wave amplitude $\Delta \mathrm{b}$ can be as large as 1-2 times the field magnitude. The magnetic field magnitude is given in the next-to-bottom panel. (The Ulysses polar passes occurred at $\mathrm{r} \sim 2 \mathrm{AU}$ from the Sun. The magnetic fields are weaker at these larger radial distances.) Note that there are many sharp magnetic field magnitude decreases that appear as spikes on this compressed timescale. These are called magnetic decreases (MDs) for obvious reasons and are associated with the nonlinear Alfvén waves (the relationship will be discussed later in this paper). The MDs are pressure-balance structures, where the plasma pressure, $\Sigma_{\mathrm{i}} \mathrm{n}_{\mathrm{i}} \mathrm{kT}_{\mathrm{i}}$, plus magnetic pressure, $\mathrm{B}^{2}$ / $8 \pi$, is constant across the structures. In the above expression for the plasma pressure, $n_{i}$ and $T_{i}$ are the densities and temperatures of the ith species (electron, proton, and ion), and $\mathrm{k}$ is the Boltzmann constant.

\subsection{Heliospheric Current Sheet Plasma Sheet (HCSPS), Corotating Interaction Regions (CIRs), and High-Speed Streams: Magnetic Storm Initial, Main, and Recovery Phases}

[10] If a solar polar coronal hole has a "finger" extending down to lower latitudes or if there is a large isolated, nearequatorial coronal hole, the high-speed solar wind emanating from these structures will interact/collide with the upstream (more distant from the Sun) slower speed stream before the streams reach 1 AU. There will be a compression of both plasma and magnetic fields in the region where the two streams interact. As previously mentioned, the intense magnetic fields created by this interaction are called corotating interaction regions (CIRs). Figure 4 is a schematic showing a coronal hole "finger" and the formation of a CIR in interplanetary space.

[11] An example of a heliospheric current sheet plasma sheet (HCSPS), a CIR, a high-speed stream proper, and the effects of all three interplanetary structures on the magnetosphere are shown in Figure 5. The top three panels are the solar wind speed, proton density, and proton temperature, respectively. The next four panels are the magnetic field components and field magnitude. The coordinate system used is GSM. In this system, $\mathbf{x}$ points radially outward from the Earth toward the Sun, $\mathbf{y}=\Omega \times \mathbf{y} /|\boldsymbol{\Omega} \times \mathbf{y}|$, where $\Omega$ is the south magnetic pole of the Earth and $\mathbf{z}$ forms a righthand system. In the figure the slow speed solar wind is at the left-hand portion and the high-speed stream is on the 


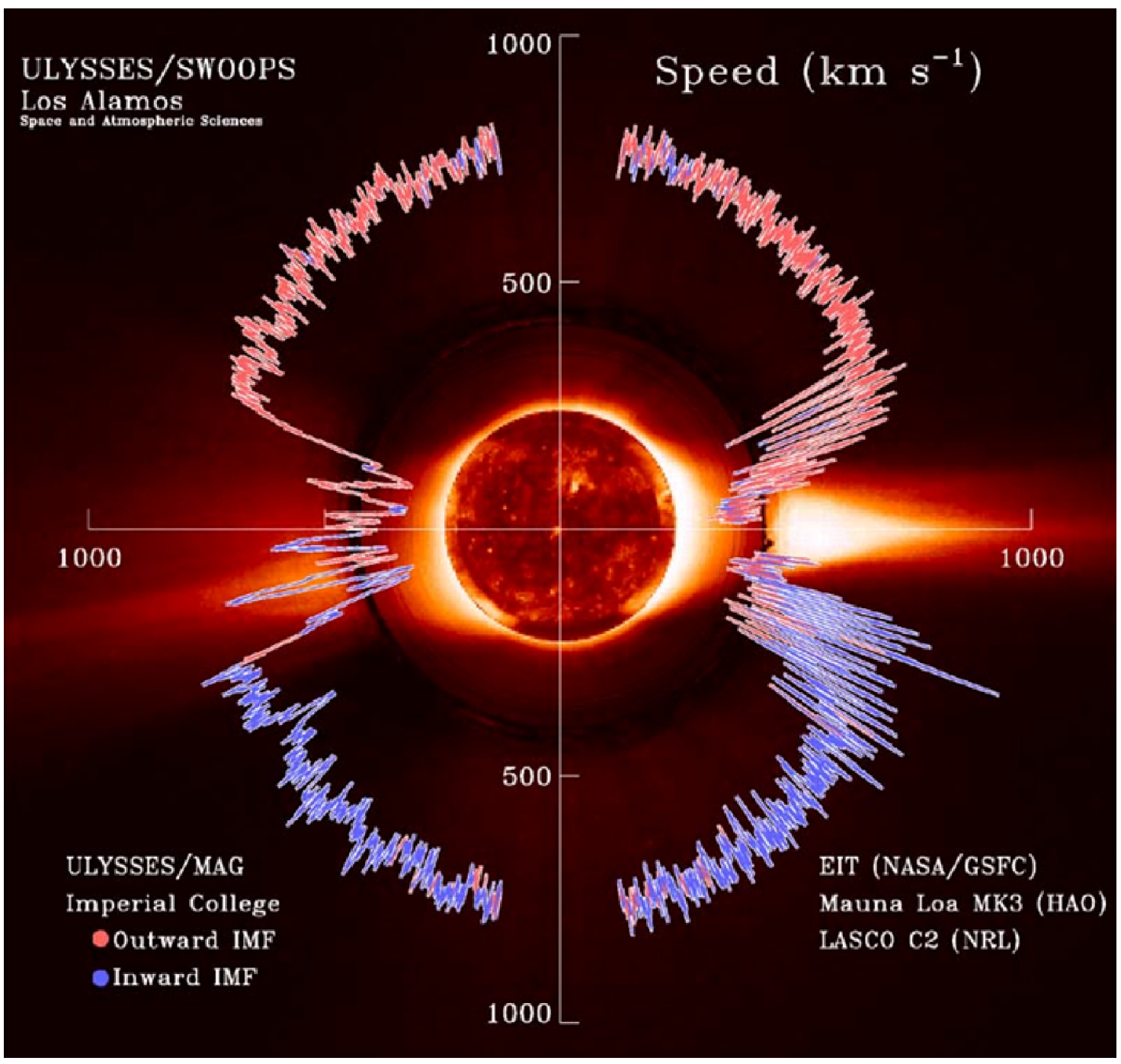

Figure 2. The solar wind speed and magnetic field polarity as a function of heliographic latitude (taken from Phillips et al. [1995]). The data were taken by the Ulysses plasma and magnetometer instrumentation during the declining phase of the solar cycle. Solar and coronal images have been added for solar wind context.

right. The "interactions" between the two streams can be noted by the high magnetic field strengths (the CIR) and the high proton temperatures near the center of the figure. It is noted that the intensity-time profiles of the enhanced magnetic field magnitudes and enhanced proton temperatures are similar to each other.

[12] The high-density HCSPS can be seen from 1800 UT on 24 January to $\sim 0700$ UT on 25 January, prior to the stream-stream compression and heating that follows. The $D_{S T}$ index is given in the bottom panel. This index is constructed from ground-based near-equatorial magnetic stations and gives magnetic effects of several external (to the Earth) magnetospheric currents: the magnetopause Chapman-Ferraro current, the magnetospheric (energetic particle) ring current, field-aligned magnetospheric currents, and the magnetic tail current (for an interesting discussion/ debate on the effect of the latter (tail) current system, we refer the reader to a series or articles: Turner et al. [2000], Baker et al. [2001], Kozyra et al. [2002], Daglis et al. [2003], and Feldstein et al. [2005]).
[13] There is a positive increase in $D_{S T}$ from 0000 to 0600 UT on 25 January. From the figure, it can be noted that this occurs at the time when the solar wind density enhancement impacts the magnetosphere. The ram pressure increase associated with this high-density, low-velocity plasma compresses the magnetosphere (both increasing the Chapman-Ferraro current intensity and moving it closer to the Earth) and causes an increase in the horizontal field strength at the surface of the Earth. This is the magnetic storm "initial phase."

[14] The physical cause/interplanetary phenomenon for the initial phase is much different than that for magnetic storms caused by fast interplanetary coronal mass ejections (ICMEs). For fast ICMEs, a fast-forward interplanetary shock forms at the antisunward edge of the interplanetary sheath, well ahead of the ICME proper. The shock compresses the slow solar wind plasma and magnetic fields, forming an interplanetary "sheath." When these enhanced plasma densities at and behind the shock impinge upon the magnetosphere, they compress it, leading to the storm 

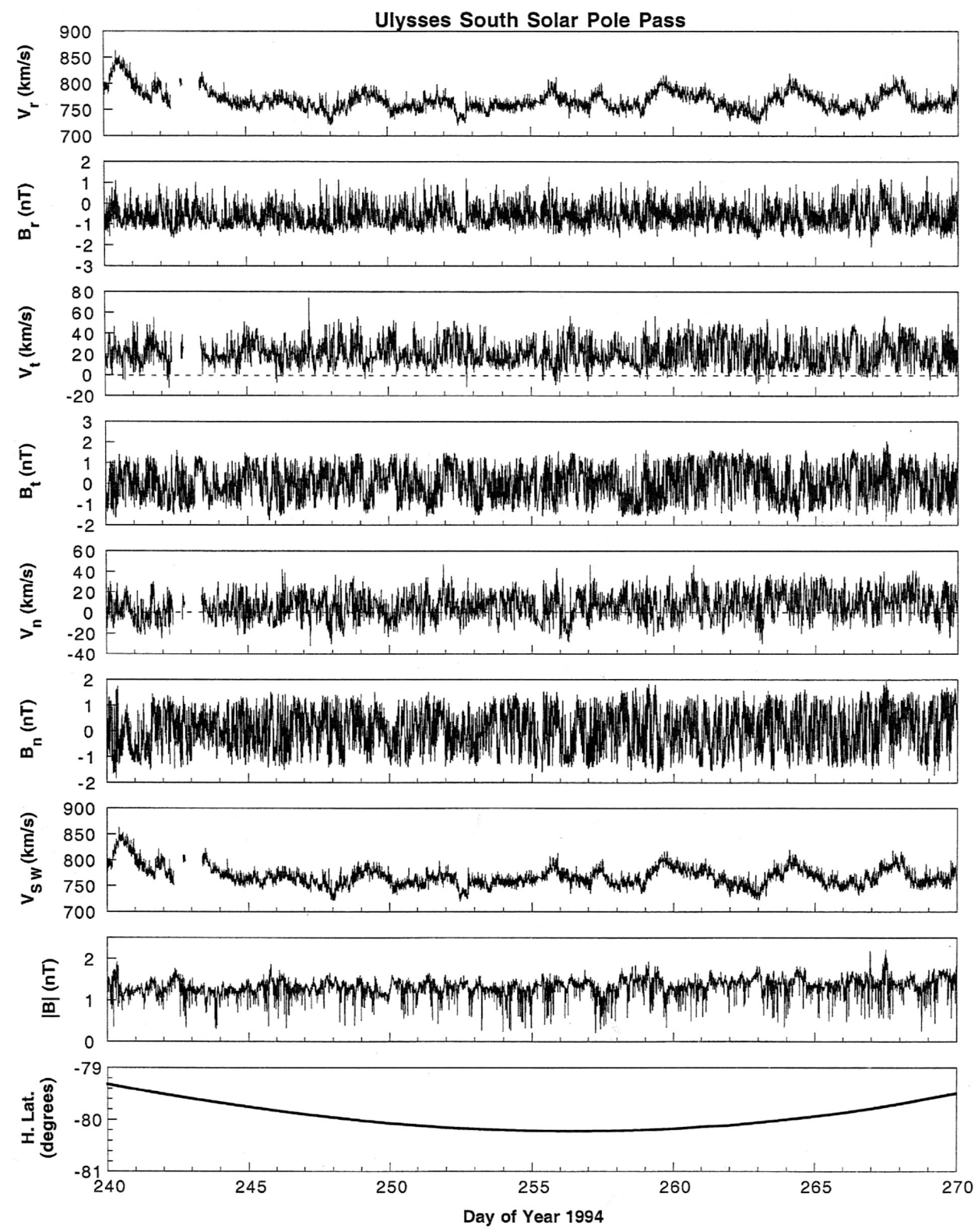

Figure 3. Large-amplitude Alfvén waves in the high-speed solar wind. The negative IMF $B_{Z}$ associated with portions of the waves are important for geomagnetic activity (if they impinge upon the magnetosphere). The magnetic decreases (negative decreases in the magnetic field magnitude) shown in the next to last panel are important for geomagnetic activity.

sudden commencement/sudden impulse (SSC/SI) and the storm initial phase that follows. However, for CIRs, as mentioned above, there are typically no fast-forward shocks formed by $1 \mathrm{AU}$, and thus there are no SSCs/SIs associated with CIR-generated magnetic storms. The presence or absence of SSCs/SIs before magnetic storm main phases may therefore give important clues as to the nature of the solar/interplanetary drivers of the events.

[15] In Figure 5, the magnetic storm main phase is present from $\sim 0500$ UT to $\sim 2100$ UT on 25 January. There are two features that are significantly different for CIR-generated storm main phases from those generated by ICMEs. CIRgenerated storms are generally only weak to moderate in intensity, $D_{S T}$ rarely being $\leq-100 \mathrm{nT}[$ Tsurutani et al.,
1995]. This event has a peak negative Dst of only $-64 \mathrm{nT}$. Second, the main phases are generally irregular in profile (the Dst indices do not typically decrease smoothly with time, as is the case of most magnetic cloud-generated storms). The causes for both of these CIR-generated storm effects can be found in the sixth (from the top) panel of the figure, that of the interplanetary $\mathrm{B}_{\mathrm{z}}$ component. Although the peak magnetic field intensity reaches values greater than $\sim 20 \mathrm{nT}$, the interplanetary $\mathrm{B}_{\mathrm{z}}$ component is highly variable. Assuming that magnetic reconnection is the process of energy transfer from the solar wind to the magnetosphere, these fluctuations lead to sporadic and weak plasma injections into the ring current and therefore a weak magnetic storm intensity. 


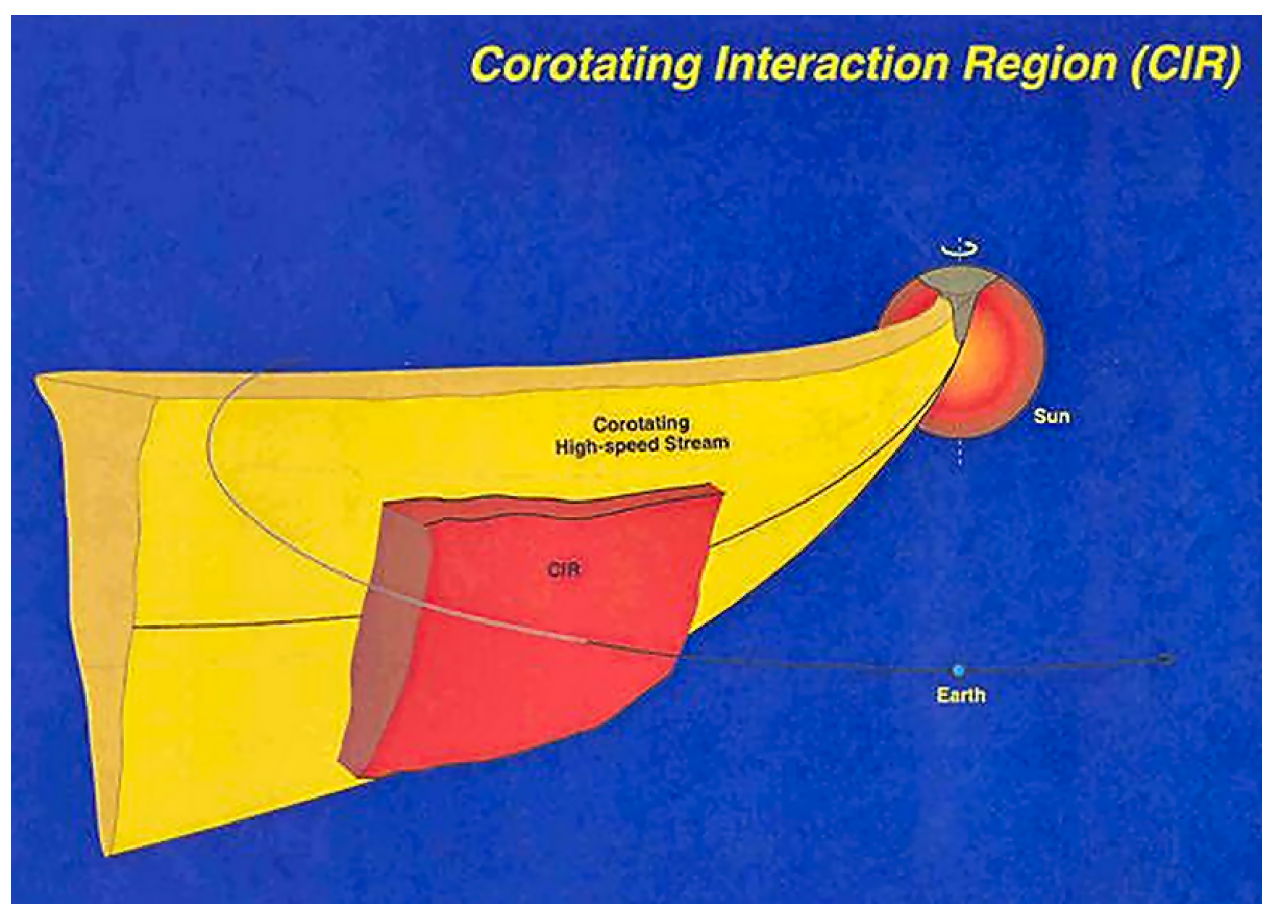

Figure 4. A schematic of a "corotating" high-speed solar wind stream emanating from a coronal hole. The interaction between the high-speed stream and the upstream slow speed stream forms a Corotating Interaction Region, or CIR. CIRs are characterized by enhanced magnetic field magnitudes and plasma temperatures.

[16] In this example, the CIR terminates abruptly at $\sim 1930$ UT on 25 January (denoted by a vertical dashed line). This has been identified as a fast reverse shock. Reverse shocks are present $\sim 20 \%$ of the time at the trailing edges of CIRs at $1 \mathrm{AU}$, so this event is somewhat unusual. The decrease in the magnetic field magnitude across the shock, and thus decrease in the IMF $\mathrm{B}_{\mathrm{z}}$ amplitudes, causes a decrease in the sporadic magnetic field reconnection rates. This decrease in reconnection rates led to the onset of the storm recovery phase.

[17] The CIR-generated magnetic storm recovery phases are likewise considerably different from those of ICMEinduced magnetic storm recovery phases. For the latter, the loss of ring current particles through physical processes such as wave-particle interactions, Coulomb collisions, charge exchange, and convection, takes place in $\sim 7-$ 10 hours [Kozyra et al., 1997] (however, see comments in section 3 of this paper). On the other hand, for CIR storm recovery phases (Figure 2), it can be noted that the $D_{S T}$ index ranges from -20 to $-40 \mathrm{nT}$ for all 24 hours of 26 January (and beyond, not shown). The CIR-generated magnetic storms appear to have very long "recovery" phases.

\subsection{High-Intensity Long-Duration Continuous AE Activity}

[18] An example of a long storm "recovery" phase following a CIR-induced magnetic storm main phase is shown in Figure 6. A 4-day interval is shown in the figure. The Dst value (bottom panel) varies from $\sim-10 \mathrm{nT}$ to $\sim-50 \mathrm{nT}$ throughout this interval. The top four panels are the solar wind velocity, proton density, magnetic field magnitude, and IMF $\mathrm{B}_{\mathrm{z}}$ component (in GSM coordinates). One of the most striking features in the figure is the correlation between the negative components of the interplanetary magnetic field $\mathrm{B}_{Z}$ (shaded) and the deceases in $D_{S T}$. There is a one-to-one relationship.

[19] The AE index is shown as the fifth panel. For every major IMF negative $B_{z}$ interval, there is a corresponding $A E$ increase.

[20] The AE value is high over the entire 4 days shown. To distinguish these events from other possible geomagnetic activity, they have been called High-Intensity LongDuration Continuous AE Activity (HILDCAA) events [Tsurutani and Gonzalez, 1987]. The strict definition is that the peak AE intensity is $>1000 \mathrm{nT}$, that the event lasts for longer than 2 days, and that the $\mathrm{AE}$ indices do not decrease below $200 \mathrm{nT}$ for over 2 hours. This is one such event.

[21] Figure 7 shows an interval of geomagnetic activity outside of a magnetic storm main phase, which is similar to a HILDCAA event but does not meet the strict criteria stated previously. The AE index is continuously high from $\sim 1600$ to 2000 UT. The peak AE value reaches $\sim 500 \mathrm{nT}$. The large (negative) AL values and $-\mathrm{AL} / \mathrm{AU}$ ratios indicate that these perturbations are not simply due to DP2 currents [Nishida, 1968a, 1968b] and also not convection bays [Pytte et al., 1978; Sergeev et al., 1996].

[22] Figure 8 shows POLAR UVI images of the northern polar regions of the Earth at the same time as the high $\mathrm{AE}$ interval of Figure 7. These images were taken in the LymanBirge-Hopfield (long) wavelength band at a cadence of $\sim 3$ min. Auroral substorms can be easily identified in the imaging data. Using the original Akasofu [1964] criteria of an auroral substorm, we have identified six substorm 
24 - 26 JANUARY, 1974 DAY 024 - 026

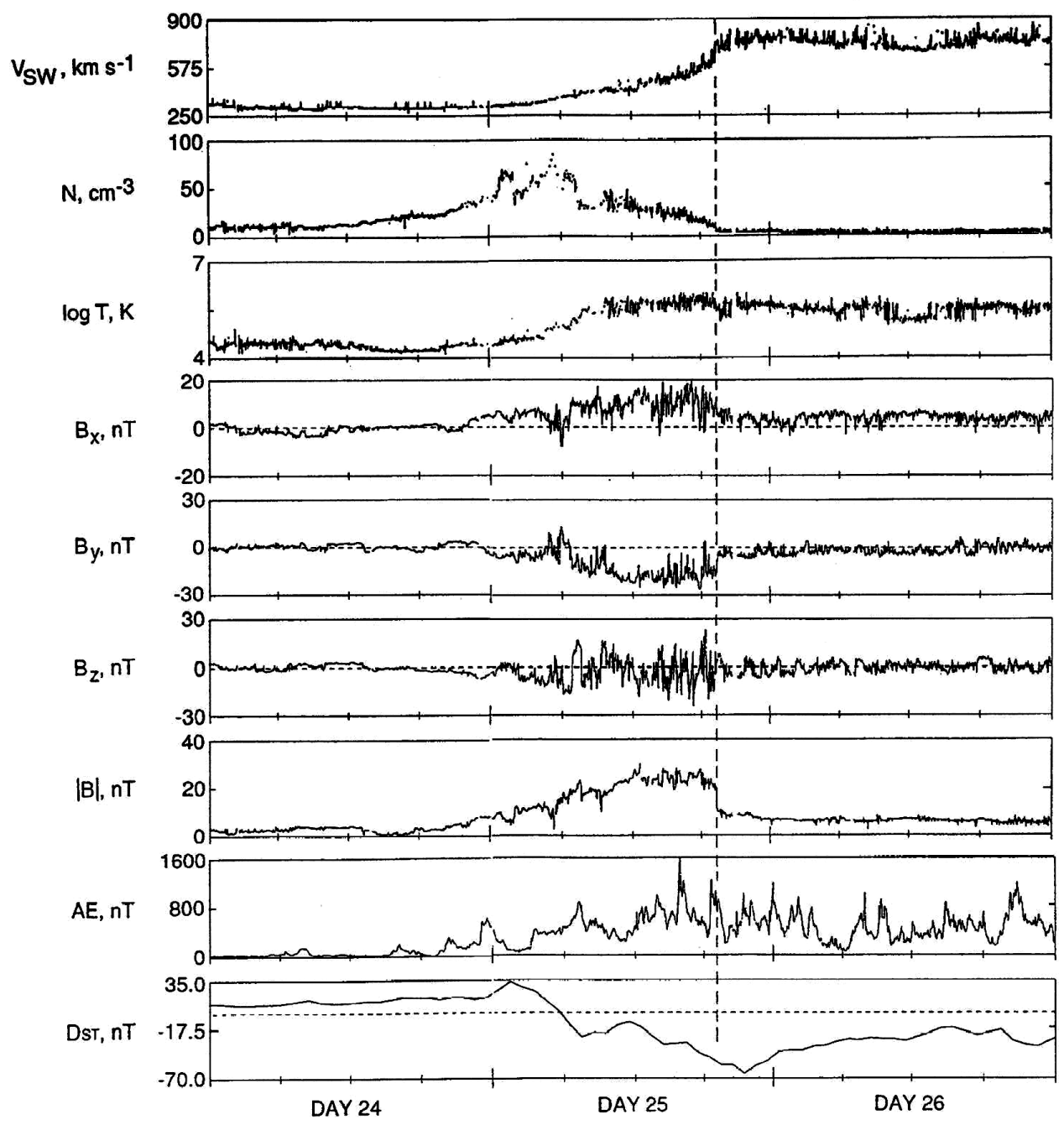

Figure 5. The heliospheric current sheet plasma sheet (HCSPS), a CIR, high-speed stream proper in IMP-8 interplanetary data just upstream of the Earth. The above three interplanetary features are responsible for the magnetic storm initial phase, main phase, and "recovery" phase, respectively.

expansion phase events occurring in the interval from 1447 to 1925 UT. These substorm intervals are indicated by shading in Figure 7.

[23] In Figure 7 it is clear that there is not a simple one-toone relationship between $\mathrm{AE}$ increases and substorms. Many of the peaks in the AE indices lie outside substorm intervals. Tsurutani et al. [2004] and Guarnieri [2005] indicate that HILDCAAs are not substorms. Guarnieri [2005] finds that during HILDCAA events there are lowintensity auroras over the entire auroral oval, from dayside to nightside. Thus there are strong indications that the solar wind-magnetosphere interaction is a complex, global one during these events. Further research on this topic is warranted.

[24] Figure 9 shows the NOAA energetic particle data during two 1998 HILDCAA events [Soraas et al., 2004]. Both events illustrate that proton injections occur in the nightside portion of the magnetosphere but that the injections are shallow and only penetrate to a minimum of $\mathrm{L} \sim$ 4.0. This is consistent with the interplanetary drivers being associated with sporadic, relatively short-duration negative IMF $B_{z}$ (Alfvénic) intervals, as discussed earlier. This has also been discussed by Sandanger et al. [2005]. Soraas et al. [2005] have noted that in spatial regions where protons should be unstable to plasma instabilities, the precipitating protons are associated with an increased loss of relativistic electrons (to be discussed later). They argue that this is consistent with the generation of Pc1 and IPDP electromagnetic waves by the unstable protons [see Cornwall et al., 1970] and the (parasitic) pitch angle scattering and loss of relativistic electrons via interaction with the electromagnetic ion cyclotron waves [Thorne and Kennel, 1971]. Cornwall et al. [1971] have suggested the formation of SAR arcs associated with the proton loss process.

[25] The bottom panel of Figure 10 gives a summary of a magnetic storm caused by a CIR. The storm initial, main and recovery phases (and their interplanetary causes) have already been described previously. The profile of a storm caused by an ICME (in particular, the magnetic cloud portion of an ICME) is also shown at the top for contrast. 


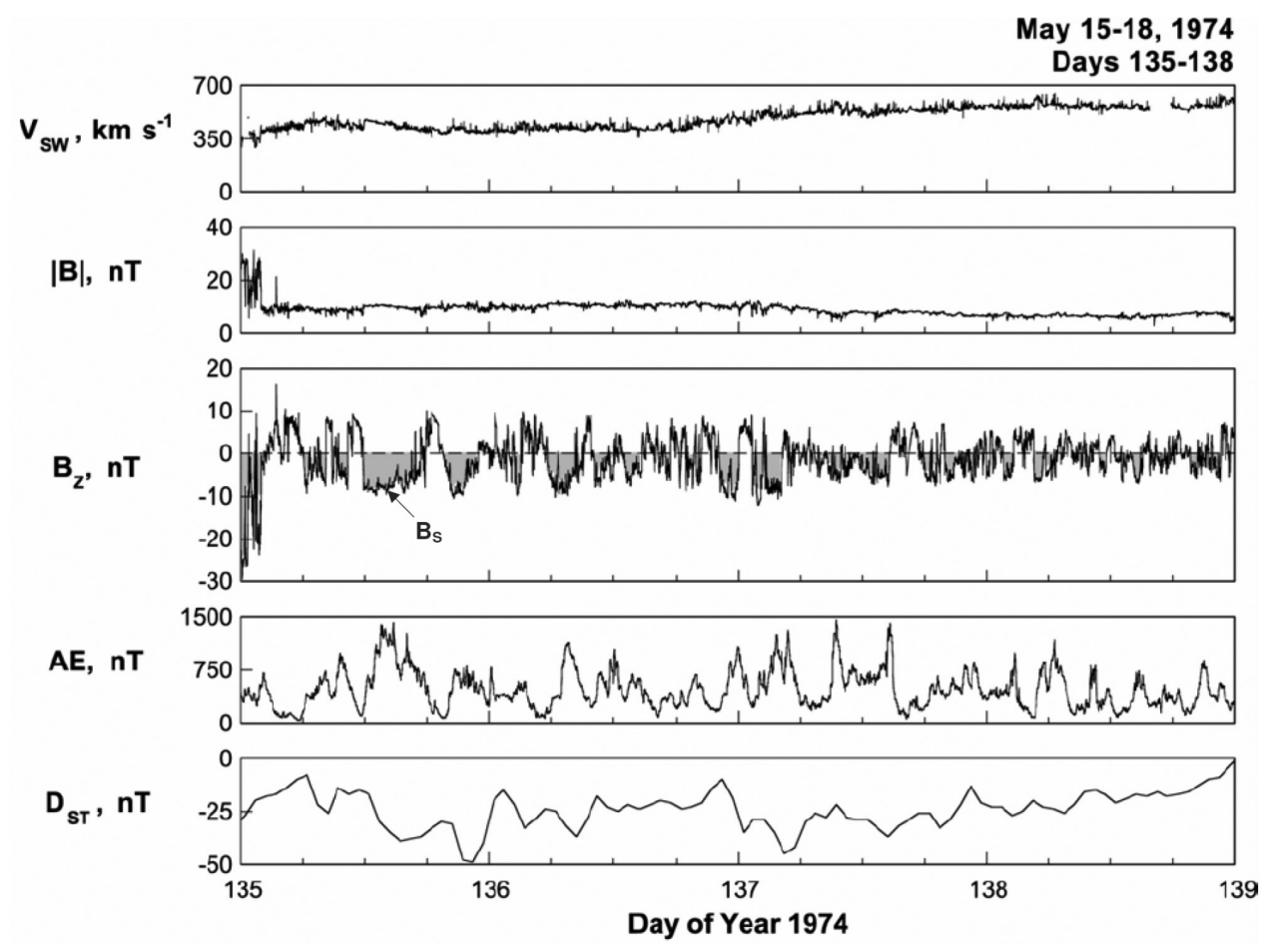

Figure 6. A magnetic storm "recovery" phase following a CIR-induced magnetic storm main phase. There is a one-to-one relationship between negative IMF $\mathrm{B}_{\mathrm{Z}}$ intervals (shaded), Dst decreases, and $\mathrm{AE}$ increases.
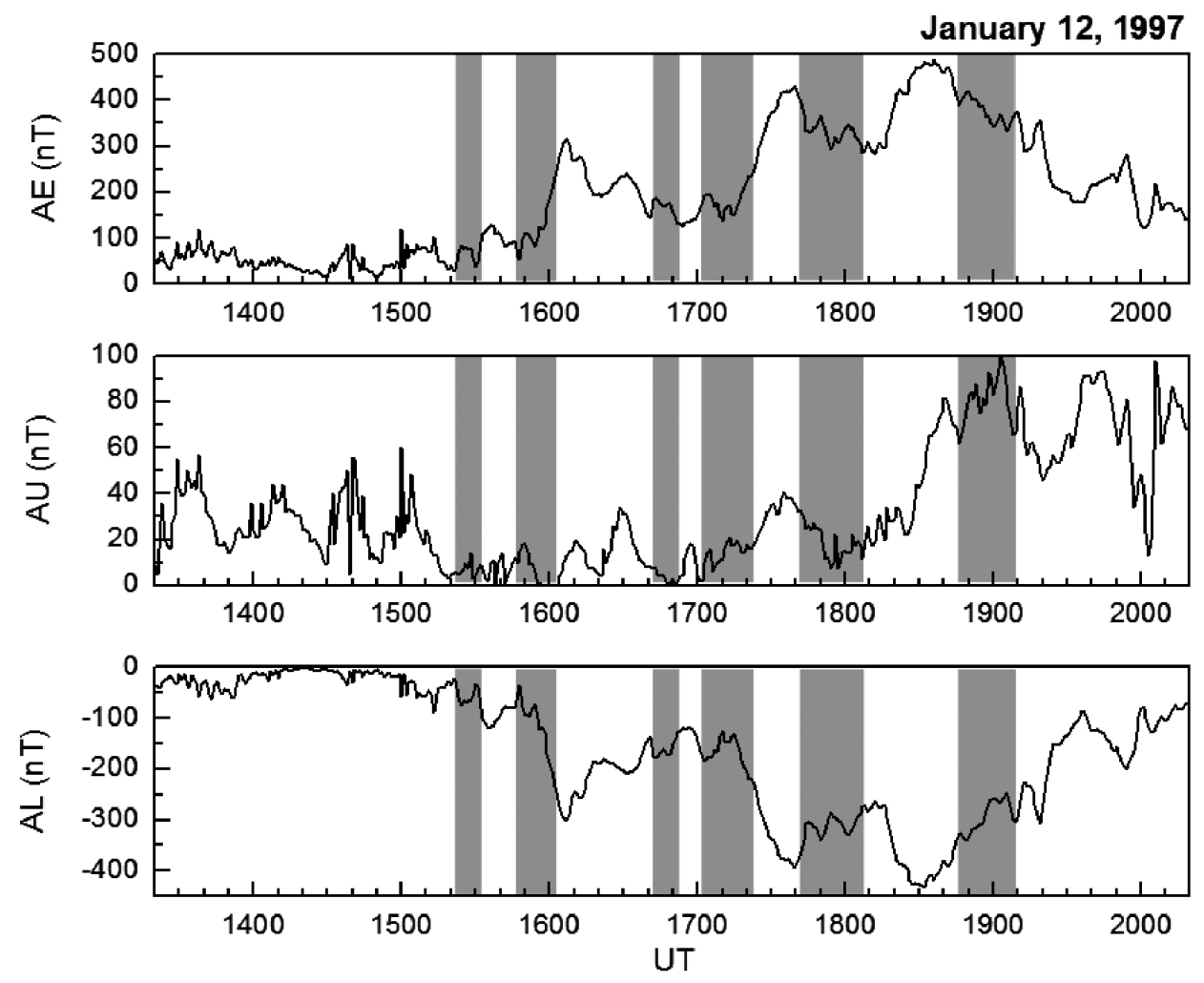

Figure 7. The AL, AU, and AE indices for a HILDCAA-like interval. Auroral substorm intervals are indicated by shading. There is no direct correspondence between substorms and the geomagnetic indices. 
1447:43 UT 1450:47 UT 1453:51 UT 1456:55 UT 1459:59 UT 1503:03 UT 1506:07 UT 1509:11 UT $1512: 15$ UT

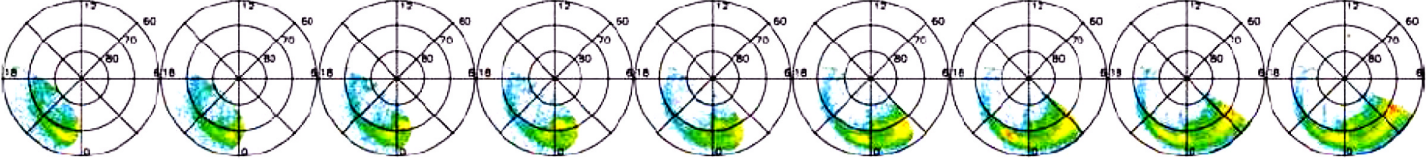

1515:19 UT 1518:23 UT 1521:27 UT 1524:31 UT 1527:35 UT 1530:39 UT 1533:43 UT 1536:47 UT 1539:51 UT

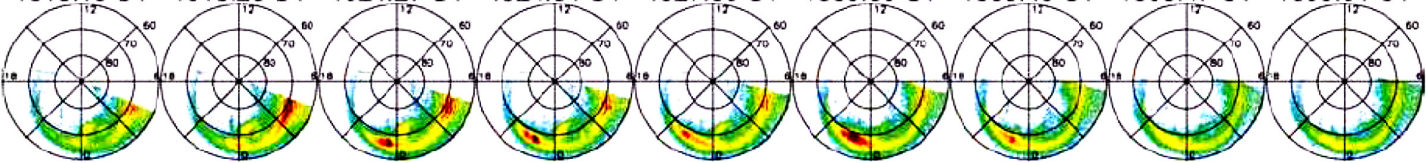

1542:55 UT 1545:59 UT 1549:03 UT 1555:11 UT 1558:15 UT 1601:19 UT 1604:23 UT 1607:27 UT 1610:31 UT

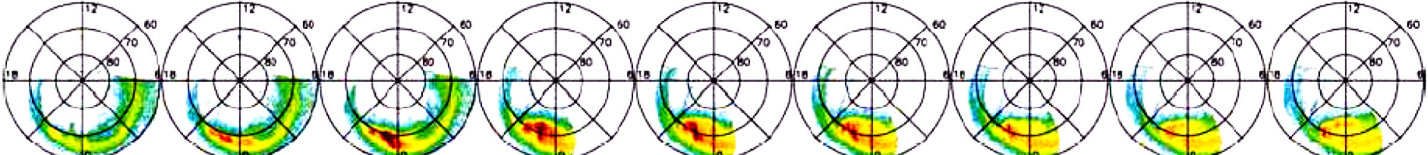

1613:35 UT 1616:39 UT 1619:43 UT 1622:47 UT 1625:51 UT 1628:55 UT 1631:59 UT 1635:03 UT 1638:07 UT

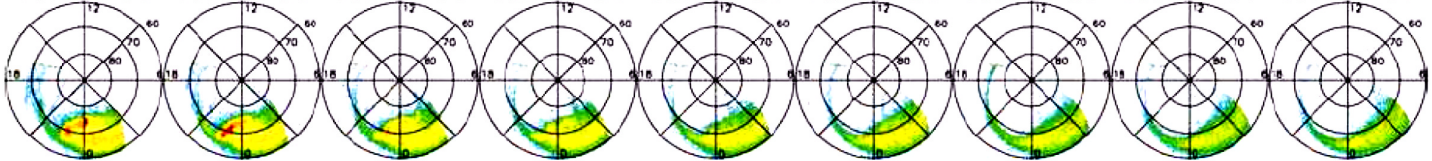

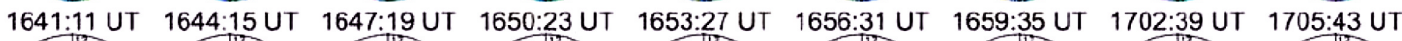

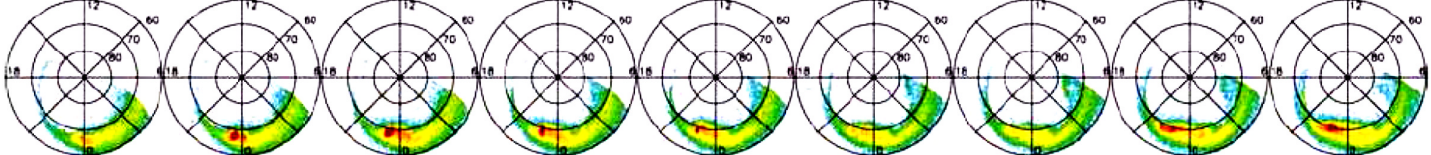

1708:47 UT 1711:51 UT 1714:55 UT 1717:59 UT 1721:03 UT 1724:07 UT 1727:11 UT 1730:15 UT 1733:19 UT

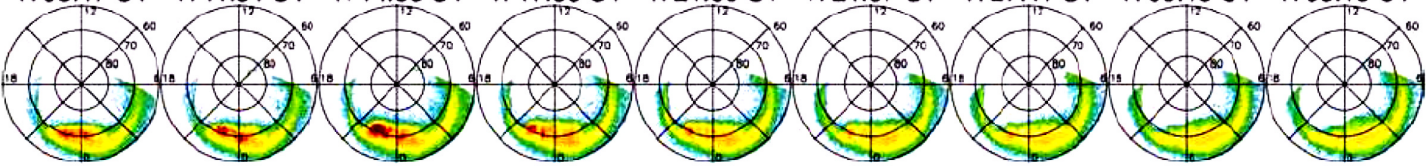

1738:14 UT 1741:18 UT 1744:22 UT 1747:26 UT 1750:30 UT 1753:34 UT 1756:38 UT 1759:42 UT 1802:46 UT

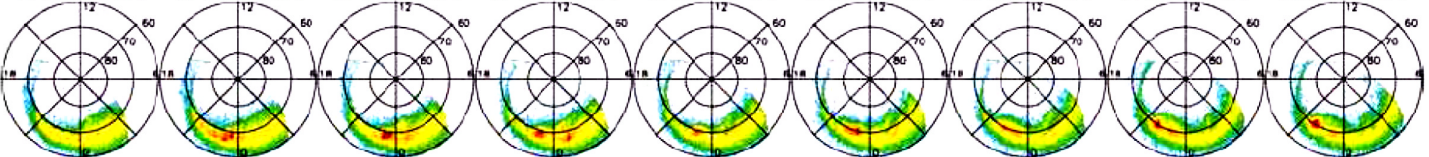

1805:50 UT 1808:54 UT 1811:58 UT 1815:02 UT 1818:06 UT 1821:10 UT 1824:14 UT 1827:18 UT 1830:22 UT

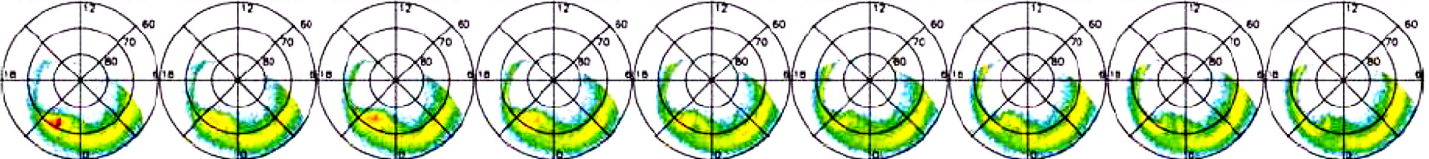

1833:26 UT 1836:30 UT 1839:34 UT 1842:38 UT 1845:42 UT 1848:46 UT 1851:50 UT 1854:54 UT 1857:58 UT

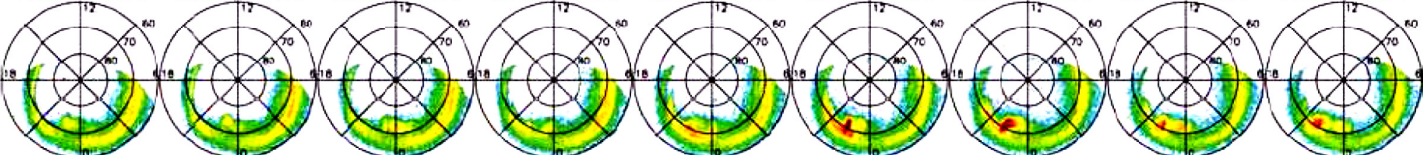

1901:02 UT 1904:06 UT 1907:10 UT 1910:14 UT 1913:18 UT 1916:22 UT 1919:26 UT 1922:30 UT 1925:34 UT

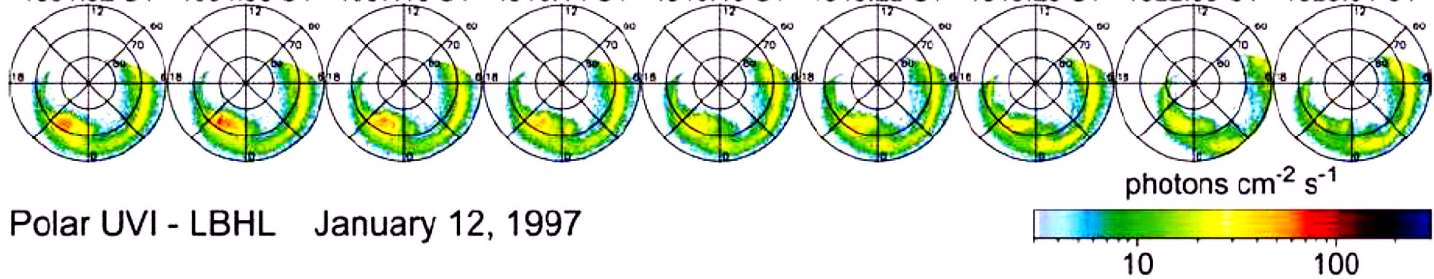

Figure 8. Polar UV images of the northern polar region. The images correspond to the time interval of Figure 4. Six intervals of substorms (as described by Akasofu [1964]) can be noted in these images and have been indicated by shading in Figure 7. 

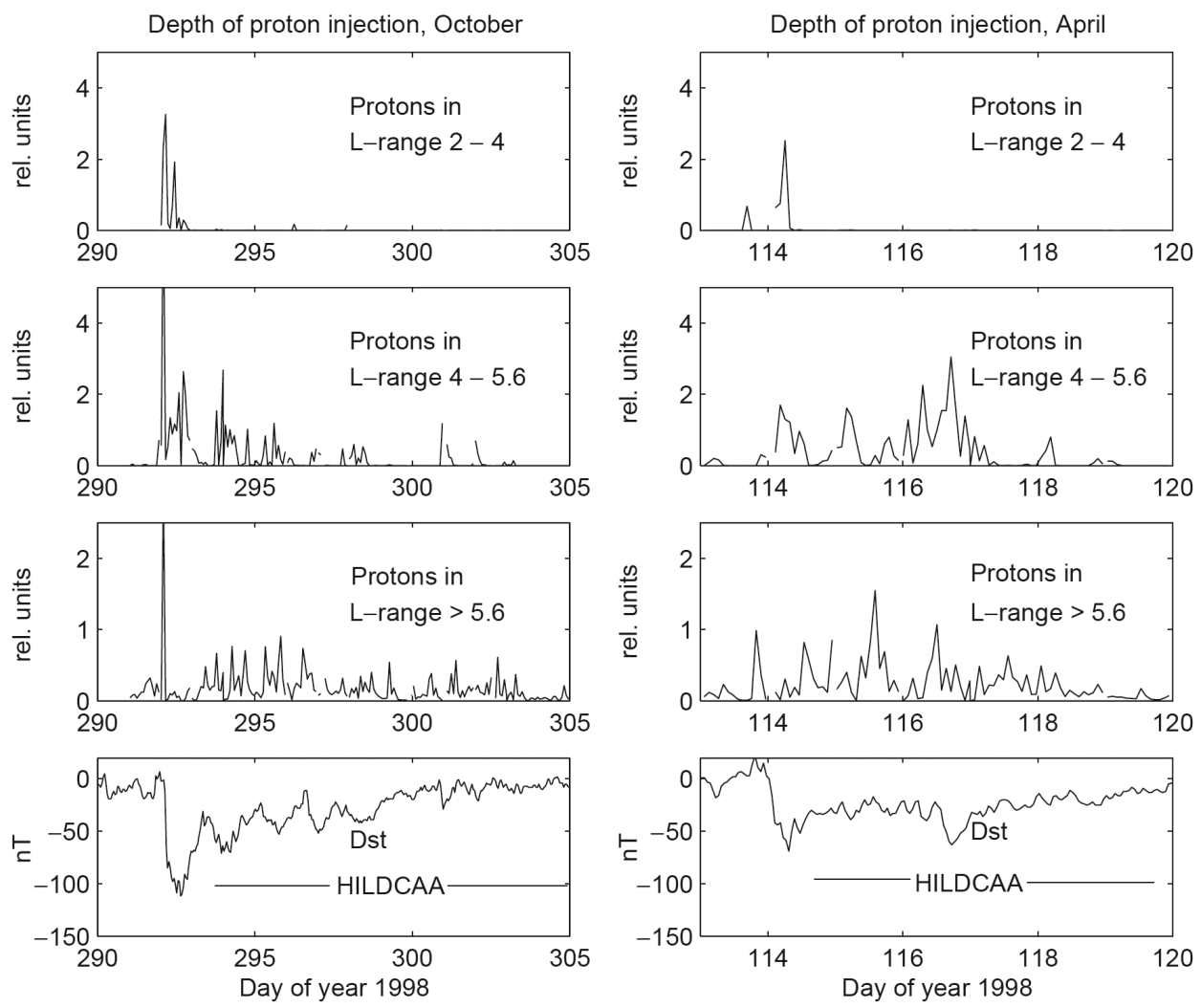

Figure 9. NOAA energetic particle data during two 1998 HILDCAA events. Protons are injected into the nightside magnetosphere to distances $>\mathrm{L}=4.0$ during these events.

We refer the reader to Gonzalez et al. [1994] and Kamide et al. [1998] for reviews of magnetic storms caused by ICMEs and storm-substorm relationships.

\subsection{Trailing Regions of High-Speed Streams and Geomagnetic Quiet}

[26] Figure 11 shows a schematic of the solar wind and resultant geomagnetic activity before and during a highspeed stream interval. At a distance of $\sim 1 \mathrm{AU}$, high-speed streams generally expand in toward the heliospheric current sheet (HCS) and its plasma sheet (superradial expansion). This may be why earlier studies with low-resolution data speculated that the HCS or "sector boundary" crossings caused geomagnetic activity [Wilcox and Scherrer, 1972]. With higher-resolution data (see Figure 5), it is noted that it is actually the negative IMF $B_{z}$ within CIRs that lead to Dst decreases and AE increases. The HCS and the HCSPS are not the causes of geomagnetic storm main phase activity per se.

[27] The conditions for geomagnetic quiet are also shown in Figure 11. Prior to the HCS crossing, the solar wind velocity is low (often as low as $\sim 300 \mathrm{~km} / \mathrm{s}$ ), the magnetic field magnitude is low, typically $<3 \mathrm{nT}$, and the Alfvénic wave relative amplitudes are low as well. Because all three of these conditions (low solar wind speeds, low B magnitudes, and low fluctuations amplitudes (or very low $B_{z}$ values)) are present at the same time, the rate of reconnection at the dayside magnetosphere is exceptionally low and resultant geomagnetic activity (AE) is at a minimum. This is an interval of geomagnetic quiet.
[28] Although such conditions can also be present during solar maximum intervals, another mechanism is more dominant for quiet intervals during that phase of the solar cycle. Magnetic clouds [Klein and Burlaga, 1982] (and their upstream sheaths) have been cited as one of the dominant causes of intense storms [Tsurutani et al., 1988; Farrugia et al., 1997]. Magnetic clouds are believed to be giant flux ropes because of their strong north-then-south $\mathrm{B}_{\mathrm{z}}$ configurations (or vice versa) [Lepping et al., 1990, 2006]. It is the magnetic cloud $\mathrm{B}_{\text {south }}$ fields that lead to the main phase of the magnetic storms via reconnection. However, the other half of the magnetic clouds, the strong northward IMFs can cause exceptional geomagnetic quiet [Tsurutani and Gonzalez, 1995]. Presumably, magnetic reconnection may be taking place at the poleward edge of the dayside cusp, but this apparently leads to negligible energy injection into the magnetosphere/ionosphere system [Oieroset et al., 2005].

\subsection{Solar Cycle Dependence of Magnetic Storms}

[29] Figure 12 shows the number of magnetic storms with Dst $<-100 \mathrm{nT}$ in the top panel and weak to moderate intensity storms with $-35 \mathrm{nT}<D s t<-75 \mathrm{nT}$ in the bottom panel for the years 1958 until 2005. The smoothed sunspot number is shown as a solid dark line. For these histograms, complex storms were counted as only one event. For the $-35 \mathrm{nT}<D s t<-75 \mathrm{nT}$ storms, the counting was done by computer due to the large number of events. The following selection criteria were used: the minimum interval between two separate events was 8 hours, and the minimum amplitude between a peak $D s t$ and the preceding valley was $\mid$ Dstpeak $|\geq 1.5|$ Dstvalley $\mid$. 
Solar Maximum (ICME) Storm

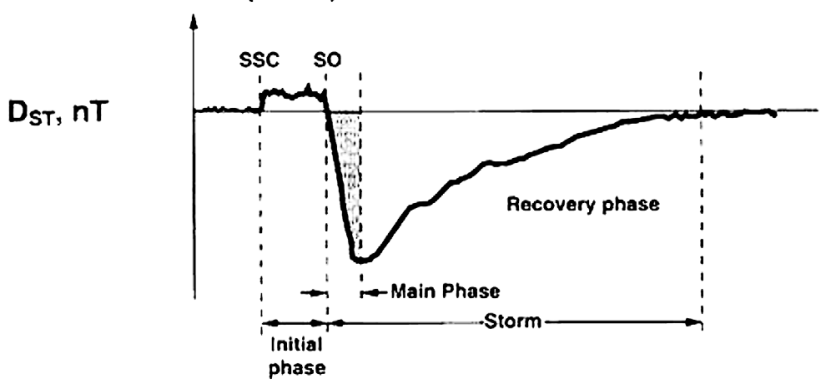

Solar Minimum (CIR) Storm

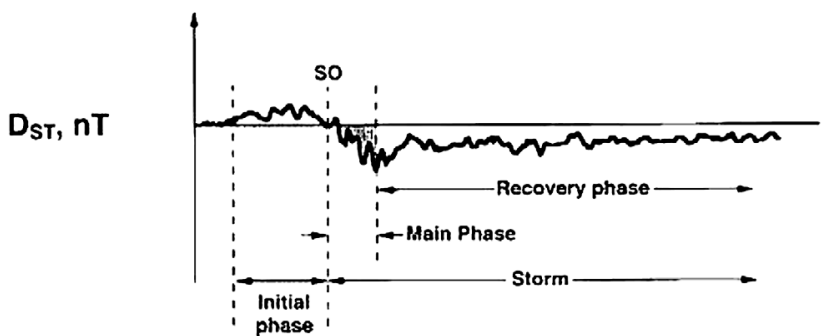

Figure 10. Schematics of magnetic storms generated by (top) ICMEs and by (bottom) CIRs. Although the profiles of these two different magnetic storms are qualitatively similar, the physical causes and characteristics of the different storm phases are different. The figure is taken from Tsurutani [2000].

[30] What is remarkable in the Dst $<-100 \mathrm{nT}$ storm histogram is the strong correlation between the number of storms and the sunspot number. The peak number of magnetic storms occurs near the solar maxima. The lowest occurrence rate of storms is found within 1 year of solar minimum for each of the four solar minima shown. The maximum to minimum number of annual storms ratio is highly variable from solar cycle to cycle, but it is typically $\sim 15$ or 20 to 1 .

[31] The solar cycle distribution of the number of $-35 \mathrm{nT}<D s t<-75 \mathrm{nT}$ intensity storms is considerably different from that of major $(D s t<-100 \mathrm{nT})$ magnetic storms [see also Gonzalez et al., 1990]. There is much less of a solar cycle dependence. The ratio of the number of storms at solar maximum to those at solar minimum is less than 2 to 1 .

[32] An interpretation of the difference between the two panels of Figure 12 is that CIRs/high-speed streams generally induce weak to moderate intensity magnetic storms, thus "filling in" the storm profiles during the declining phase and at solar minimum. An exception to this behavior should, however, be mentioned. It is observed that at the solar minimum of 1964-1965, there is a great decrease in weak to moderate intensity storms (the ratio of storm numbers at solar maximum to solar minimum reaching more than 10 to 1 ). For this particular interval, the heliospheric current sheet measured at Earth was characterized by a very well organized four sector structure [Gonzalez and Gonzalez, 1987], perhaps offering an explanation for this strong decrease of weak to moderate intensity magnetic storms.
[33] It is clear that many of the weak to moderate intensity magnetic storms are caused by ICME events and that CIRs could cause some of the major storms. A clear separation is not possible unless each and every storm is investigated for its interplanetary cause. This is clearly beyond the scope of this present effort. However, the general trend of which type of interplanetary event is responsible for what intensity magnetic storm is reasonably clear.

\subsection{Long-Term Energy Input Into the Magnetosphere/Ionosphere During the Declining Phase of the Solar Cycle}

[34] During the declining phase of the solar cycle, there is a predominance of corotating high-speed solar wind streams and an absence of ICMEs in the heliosphere. The 19731975 declining phase interval was exceptional in producing geomagnetic activity because there were two major corotating streams present at the same time. The Earth's magnetosphere was inundated by these streams twice during each $\sim 27$ day interval (it is shown by Tsurutani et al. [2005] that if the second CIR does not produce a magnetic storm main phase, it appears as if the first CIR storm has a "recovery phase" of $\sim 27$ days). The magnetosphere was thus almost continuously bathed in one or the other stream almost all of the time. The solar wind and relevant geomagnetic activity data for 1974 is shown in Figures 13 and 14. The highspeed stream velocities are given in the third panel of Figure 13. One can note that streams are almost continuously present throughout this year. The two distinct sequences

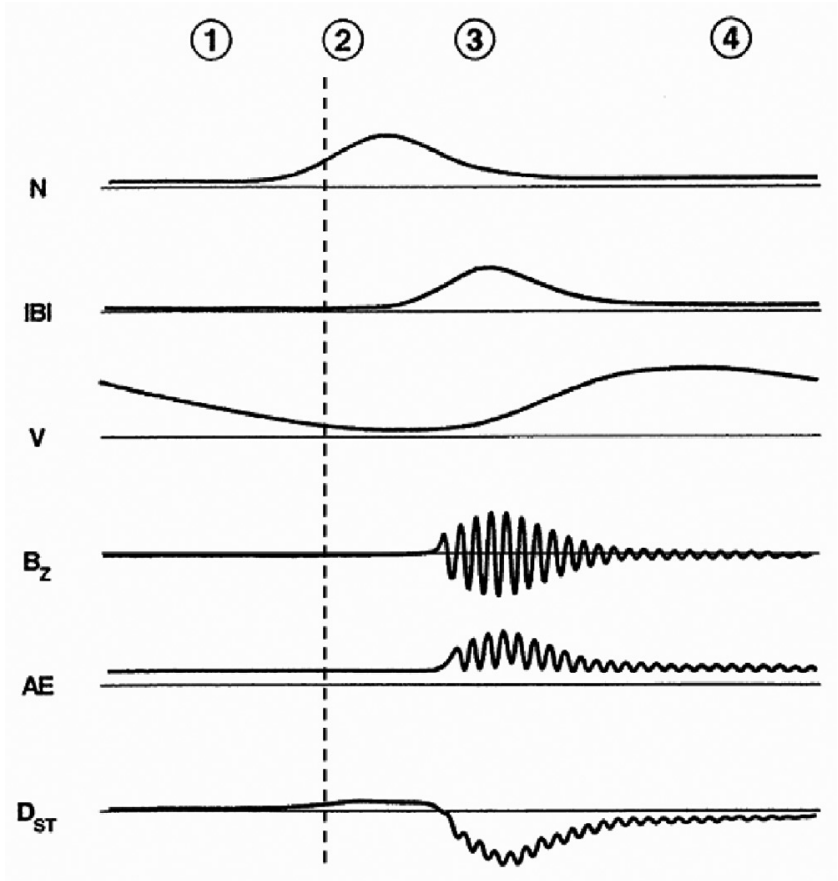

Figure 11. A schematic of a solar wind high-speed stream and related geomagnetic activity. The interplanetary conditions for different types of geomagnetic activity are indicated by numbers: (1) geomagnetic quiet, (2) a storm initial phase, (3) a magnetic storm main phase, and (4) the storm extended "recovery" phase. 

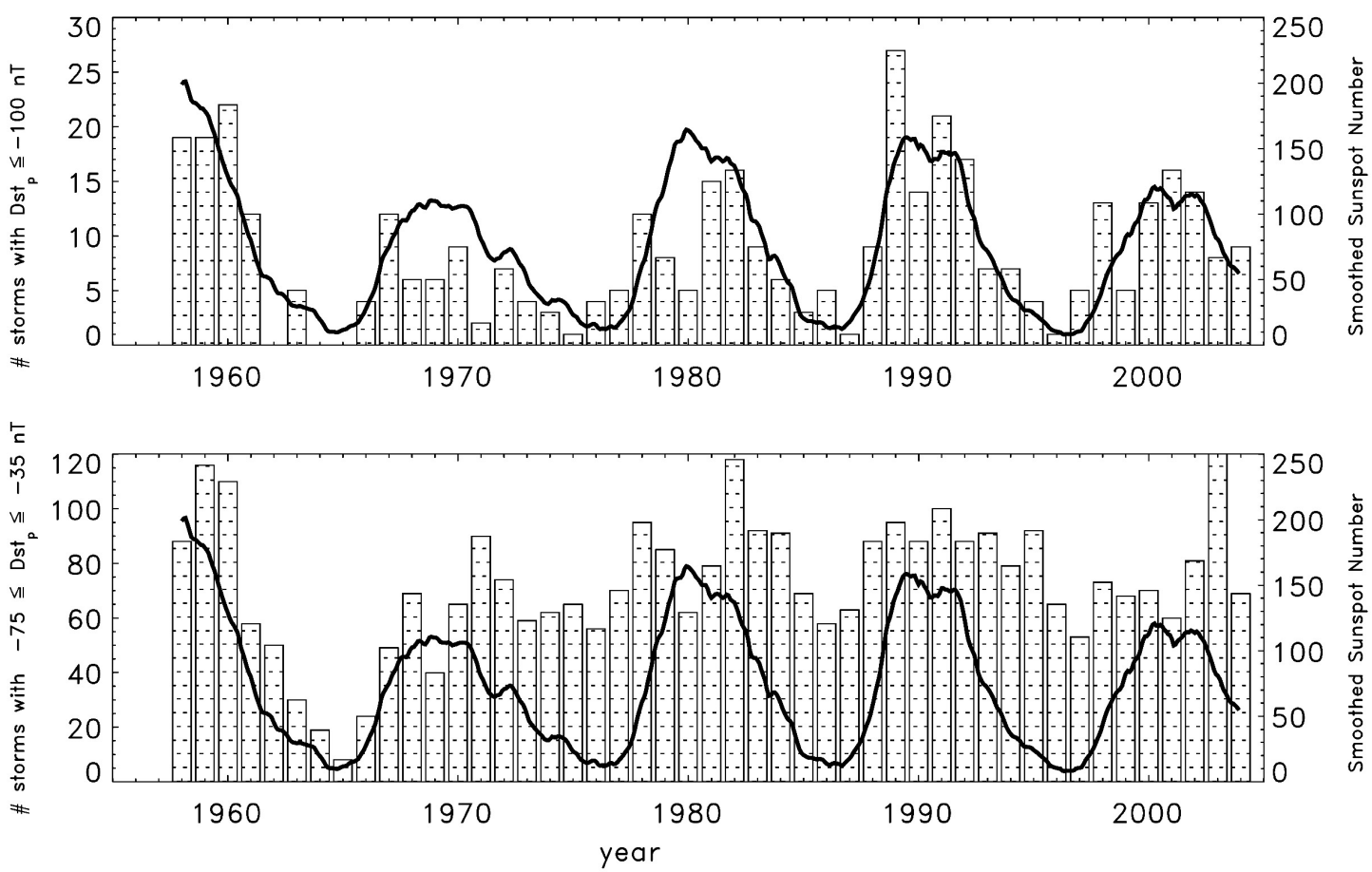

Figure 12. Occurrence of magnetic storms per year between 1958 and 2004. The number of magnetic storms/year with $D s t<-100 \mathrm{nT}$ are given at the top and those with $-35>D s t>-75 \mathrm{nT}$ on the bottom. The smoothed sunspot number is shown as a solid dark line. The number of major $(D s t<-100 \mathrm{nT})$ magnetic storms follow the solar cycle sunspot number. There are $\sim 15$ to 20 major magnetic storms/year during solar maximum and only $\sim 1$ to 2 during solar minimum. The ratio is $\sim 15$ to 20 . For weak to moderate intensity magnetic storms, there is a much smaller solar cycle dependence. CIRs/high-speed streams are presumably responsible for most of the weaker storms.

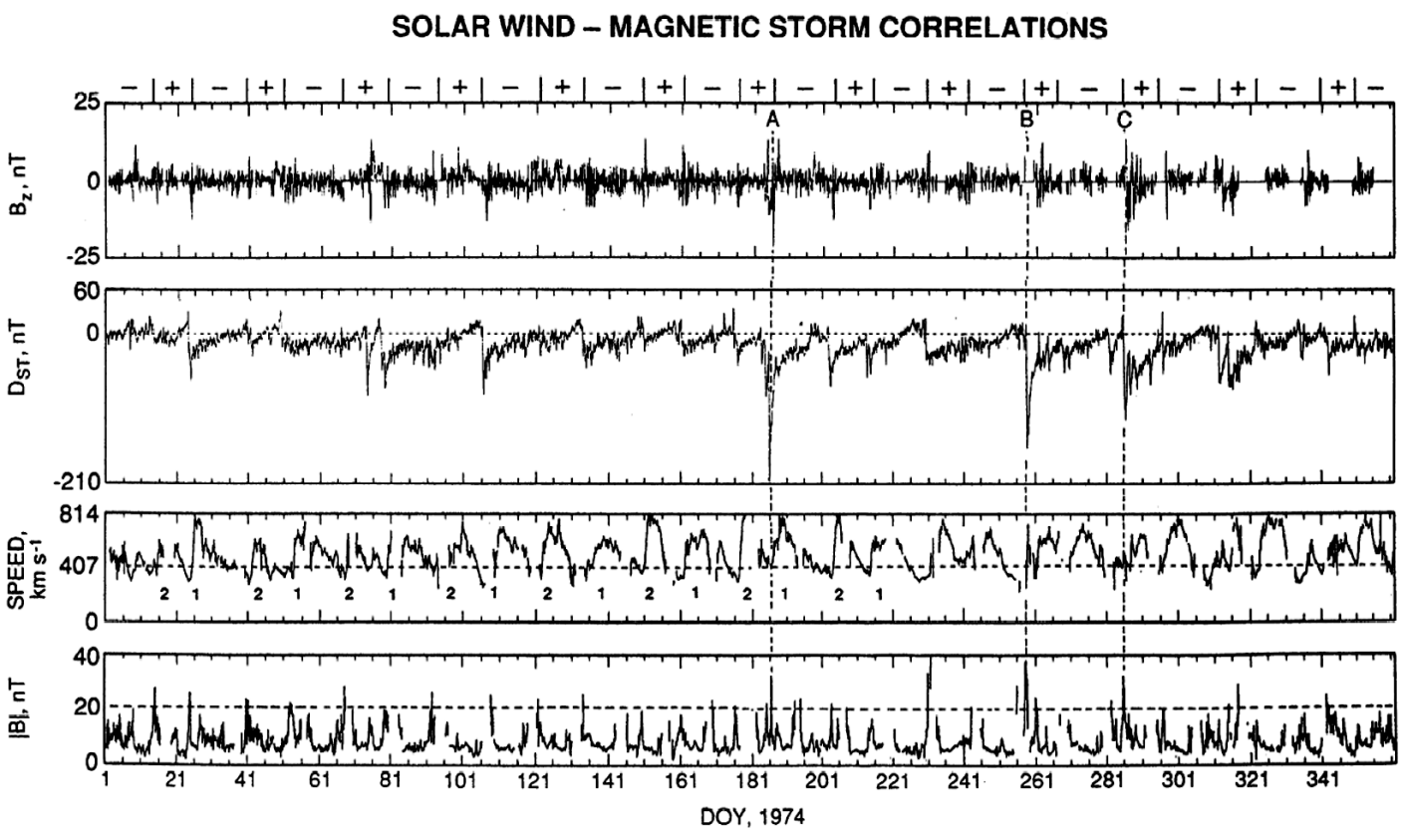

Figure 13. The solar wind and geomagnetic activity for 1974 . Two $\sim 27$ day recurrent high-speed stream sequences are indicated (third panel), sequence 1 and sequence 2. These stream-generated CIRs cause only minor to moderate magnetic storms. Because of the high-speed stream Alfvén waves, the AE indices are continuously high during the year. 


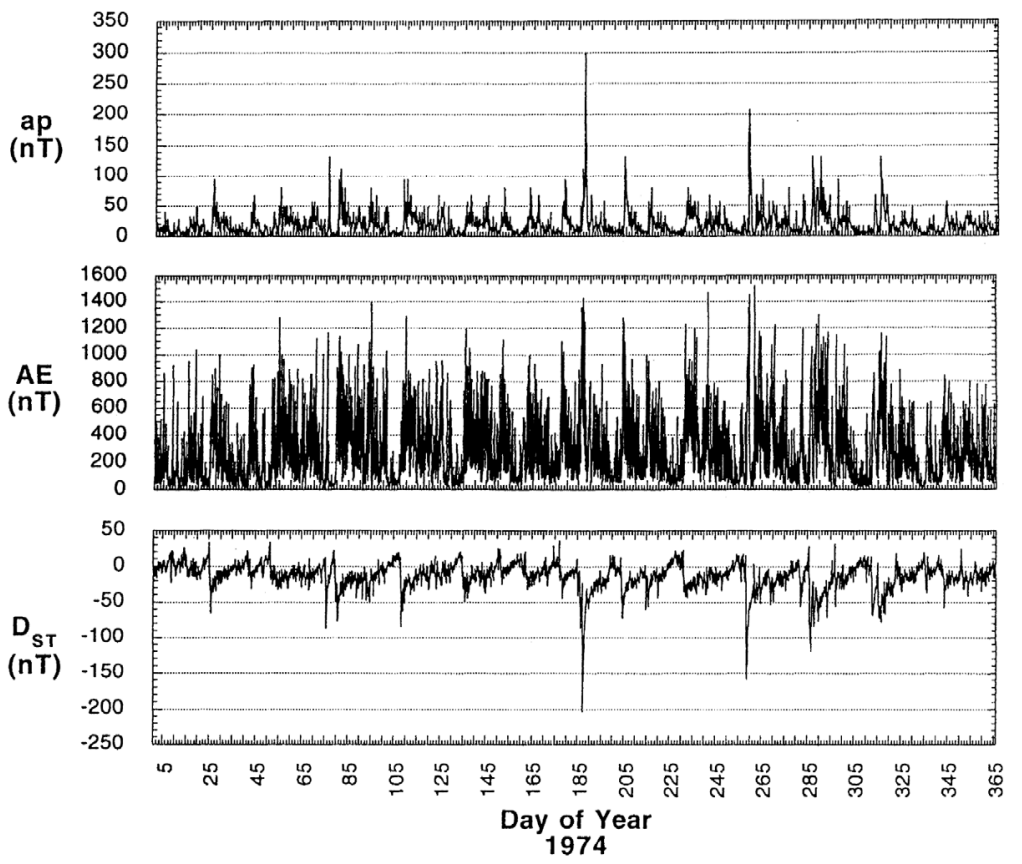

Figure 14. The Ap. AE, and Dst indice values during 1974.

of streams are labeled 1 and 2 in this panel. The other three panels, from top to bottom, contain the IMF Bz component, the Dst index, and magnetic field magnitude, respectively. The Dst values are relatively quiet throughout the year. There are only three magnetic storms with intensities Dst $<$ $-100 \mathrm{nT}$. They are labeled events A, B, and C. They have been investigated in detail and it was determined that the interplanetary causes were ICMEs for all three cases. More detail can be found in the work of Tsurutani et al. [1995]. On the scale of the figure, the CIRs appear as magnetic field magnitude positive spikes at the leading edges of the streams. If there is any magnetic storm Dst responses, it will be associated with this part of the stream (where the interplanetary magnetic field strengths are high and the potential for large negative $\mathrm{Bz}$ exists). In the interval from days 24 to 26 , the sequence 1 CIR leads to a $\sim-62 \mathrm{nT}$ storm. However, at approximately day 176, a CIR associated with an even faster solar wind stream (from sequence 2) caused a storm main phase of only $D s t \sim-48 \mathrm{nT}$. There are also some CIRs/streams that cause imperceptible Dst changes. In general, magnetic storms caused by CIRs are only weak $(-25>D s t>-50 \mathrm{nT})$ to moderate $(-50>\mathrm{nT}<$ Dst $>-75 \mathrm{nT})$ in intensity.

[35] At the very top of the panel are the interplanetary magnetic field polarities. A plus sign by convention indicates outward pointing interplanetary magnetic fields and a minus sign, inward pointing fields. The vertical bars separating the two polarity regions are the general locations of the heliospheric current sheet. It can be noted that the highspeed streams often occur just adjacent to the HCS. This point was discussed in a previous section.

[36] Near the spring and vernal equinoxes, the polarity of the interplanetary magnetic field influences the IMF $\mathrm{Bz}$ values and therefore the level of geomagnetic activity. A positive sector polarity magnetic field has positive By field components and conversely, a negative sector polarity has negative By field components. Thus during the fall, positive sectors will have enhanced negative GSM Bz values, while during spring it is the negative sectors that will have enhanced negative GSM Bz values. The results of these features can be noted in the figure. The Dst levels are slightly higher for negative sectors during spring and for positive sectors during fall. However, it is noted that geomagnetic activity is strong throughout the entire year, irrespective of season. This is due to the highly fluctuating nature of the interplanetary magnetic field in the form of nonlinear Alfven waves. The large Bz components of the waves give substantial negative values during either positive or negative sectors.

[37] Figure 14 indicates that although the CIR-caused magnetic storms were not exceptional during 1974, the AE index, taken over the whole year, was. The very longduration, high-intensity AE intervals occur in the "recovery" phase of the CIR-induced magnetic storms (compare the second and third panels). The AE values are highest at the beginning of the storm and then gradually taper off over a week or more. The general profile of the Alfvén wave amplitudes is similar in nature (but not shown here).

[38] The 2.5-min average AE indices over 1974 was $283 \mathrm{nT}$. The value during the following solar maxima (it was a dual peak solar maximum) was $221 \mathrm{nT}$ in 1979 and $237 \mathrm{nT}$ in 1981 (in the 1980 local sunspot number decrease, AE was $180 \mathrm{nT}$ ).

[39] Following the Akasofu [1981] proxy for energy input into the magnetosphere and ionosphere:

$$
\mathrm{U}_{\mathrm{T}}=\mathrm{U}_{\mathrm{O}}+\mathrm{U}_{\mathrm{A}}+\mathrm{U}_{\mathrm{J}}
$$

where $\mathrm{U}_{\mathrm{O}}$ and $\mathrm{U}_{\mathrm{A}}$ are the ring current and auroral energies, and $U_{J}$ is the rate of Joule heating. The Joule heating rate has been approximated by $2 \times 10^{15} \mathrm{AE}(\mathrm{nT}) \operatorname{ergs~s}^{-1}$ and the auroral particle energy by $1 \times 10^{15} \mathrm{AE}(\mathrm{nT}) \mathrm{ergs} \mathrm{s}^{-1}$. The ring current energy has been approximated as $\mathrm{U}_{\mathrm{O}}=4 \times$ $10^{20}(\mathrm{~d} D s t / \mathrm{dt}+D s t / \tau)$, where $\tau$ is the ring current decay 

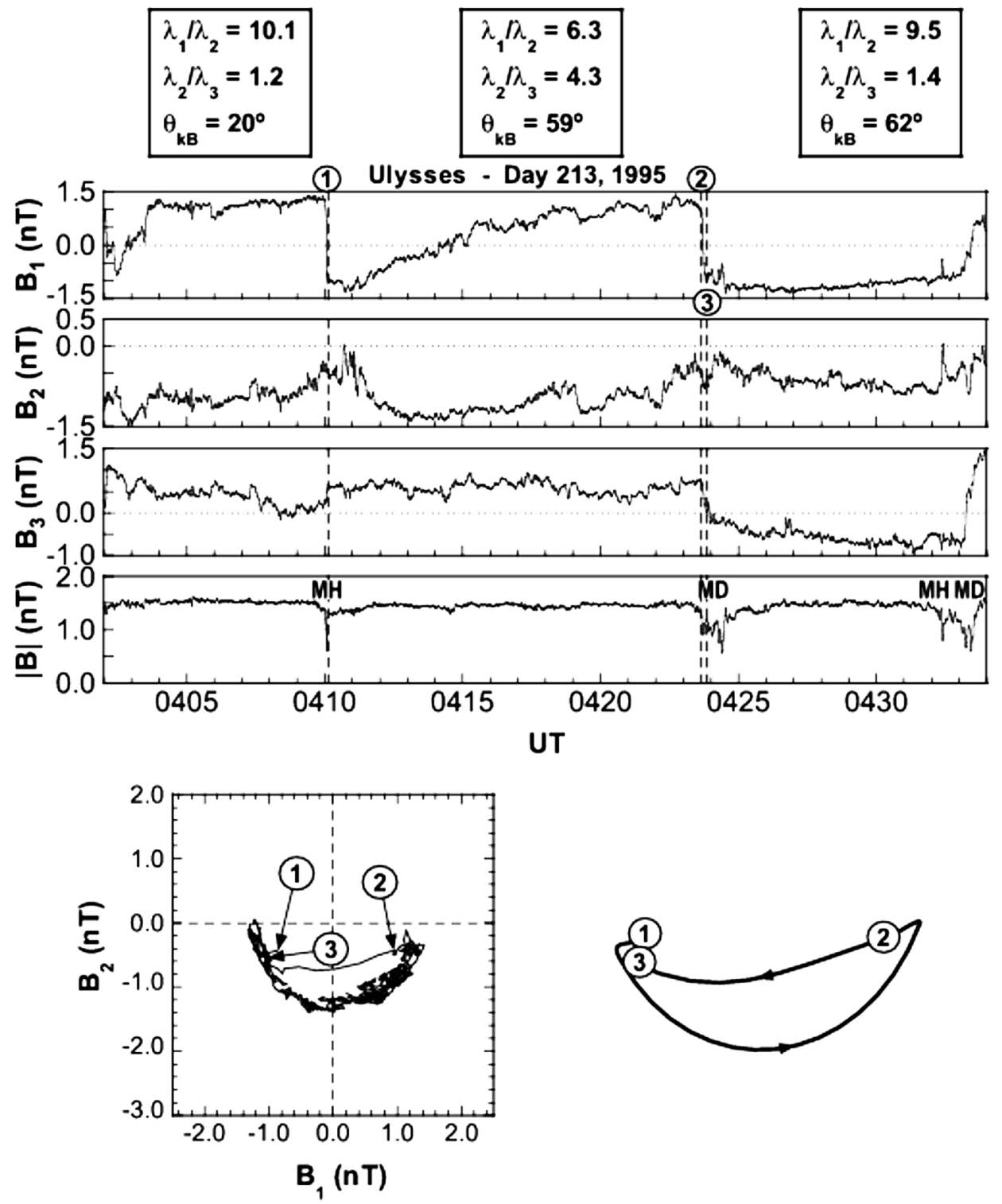

Figure 15. Three cycles of an interplanetary phase-steepened Alfvén wave. The wave phase-steepened edges are directional discontinuities that may trigger substorm expansive phase onsets. The magnetic holes (MHs) and magnetic decreases (MDs) are pressure balance structures that can lead to ram pressure increases of up to $\sim 2$ times ambient value when they impinge upon the magnetosphere.

time (note that Pulkkinen et al. [2002] has shown that the above expression for $\mathrm{U}_{\mathrm{O}}$ may be an overestimation during intense storms).

[40] It is found that there was far more energy injected during the 1974 declining phase of the solar cycle than during solar maxima for years 1979 and 1981 [Tsurutani and Gonzalez, 1997]. The actual ratio of energy input during the declining phase to that during solar maximum is presently unknown. The above expression from Akasofu [1981] [see also Pulkkinen et al., 2002] is, of course, only a proxy. The Guarnieri [2005] results that auroras are present throughout the entire auroral oval (and sometimes the polar cap as well) during HILDCAA intervals increases the amount of auroral energy deposition during the declining phase. In contrast, substorm energy deposition occurs primarily only in the midnight sector. Since the AE index is a measure of the maximum magnetic deviations, HILDCAA auroral energy deposition may be double (or more) the Akasofu [1981] proxy values (energy over the entire auroral oval, rather than just the midnight sector). A better estimate of the ratio is current work in progress.

\subsection{Interplanetary Alfvén Waves and Their Microstructure}

[41] Figure 15 shows three cycles of an interplanetary Alfvén wave. The coordinate system is the minimum variance system where 1 is the direction of the largest eigenvector of the covariance matrix, 2 is the intermediate variance direction, and 3 is the minimum variance direction. The three cycles of the wave is best noted in the top $\left(B_{1}\right)$ panel. The three cycles are present from the left-hand edge 

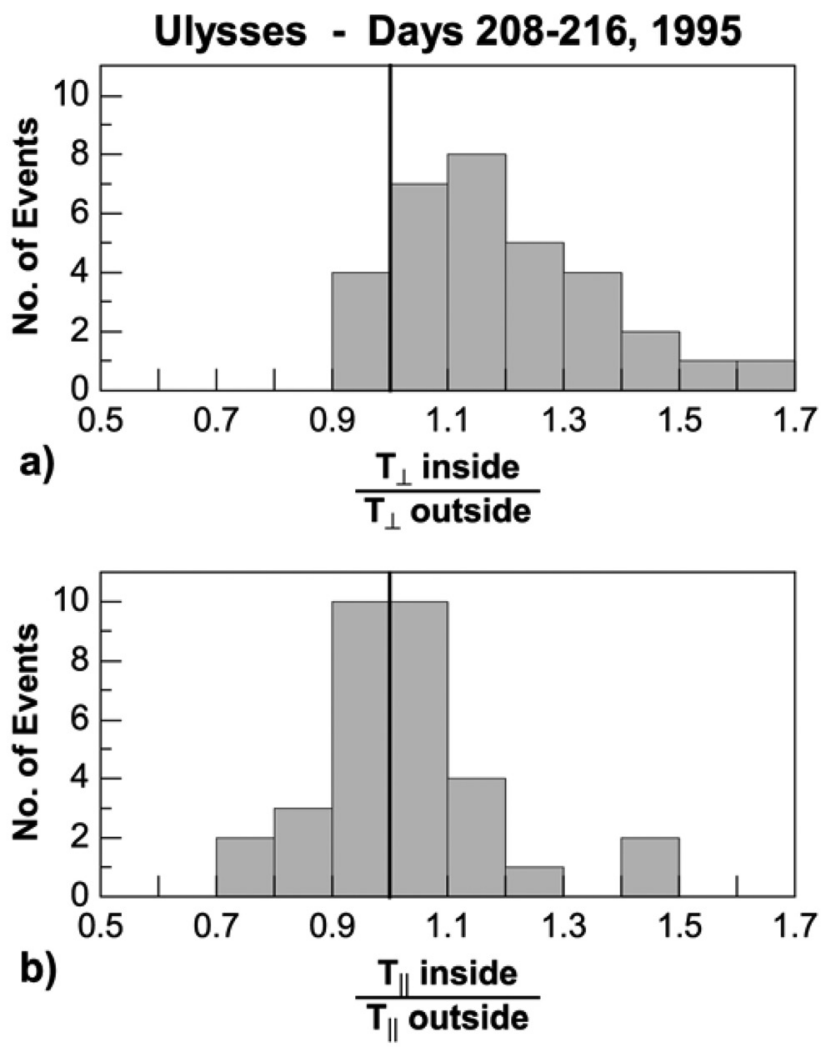

Figure 16. The ratio of proton temperatures inside MDs to that outside. The perpendicular (to the magnetic field) temperature ratio distribution is shown at the top and the parallel temperature ratio distribution is shown on the bottom. The typical ratio is greater than one for the perpendicular temperatures, indicated that local plasma perpendicular heating is occurring. Taken from Tsurutani et al. [2002].

to time 1 , from time 1 to time 3 , and from time 3 to the right-hand edge of the figure.

[42] There are several noteworthy features in the waves that are important for geomagnetic activity. First, it is obvious that the waves are far from sinusoidal. In the middle wave cycle, from times 1 to 3 , the $B_{1}$ component rotates slowly from a peak negative value at time 1 to a peak positive value at time 2 and then suddenly changes to a large negative value from time 2 to time 3 . This sharp field change in direction from time 2 to time 3 has been called a "directional" discontinuity in the literature (the direction of the magnetic field vector changes abruptly or "discontinuously" [Burlaga, 1969]). The inset at the bottom of the figure shows a hodogram of the $B_{1}-B_{2}$ magnetic field components. From time 1 to time 2, the field rotates in an arc. It rotates back in an arc from times 2 to 3 . It is argued that this is all one wave, but phase-steepened in nature (this term means that the wave rotation in phase is not smooth such as with a sinusoidal wave, but is steepened at one edge). Wave phase-steepening has been discussed theoretically for over 30 years [Cohen and Kulsrud, 1974; Medvedev et al., 1997; Medvedev, 1999; Vasquez and Hollweg, 2001]. Plasma wave phase-steepening has been experimentally shown to exist for both right-hand circularly polarized (magnetosonic or the whistler branch) and lefthand circularly polarized waves [Tsurutani et al., 1997]. Medvedev et al. [1997] have theoretically shown magnetic compression for phase-steepened right-hand waves. For interplanetary Alfvén waves, phase-steepening occurs without magnetic compression.

[43] Magnetospheric researchers have suggested that sharp interplanetary magnetic field northward turnings or southward turnings could trigger expansion phases of substorms [Iyemori, 1980; Lyons et al., 1997]. We now know what these sharp directional turnings are the phase steepened edges of Alfvén waves. There is the continuous presence of Alfvén waves in high-speed streams (Figure 3). The rate of occurrence of discontinuities can increase by a factor of 10 or more over slow stream rates. However, the imaging data shown in Figure 7 indicates there is a lack of correlation between peak $\mathrm{AE}$ values and substorm expansion phases. In fact, the majority of HILDCAA auroras are somewhat featureless. Is it possible that the conditions for HILDCAAs suppress the onsets of substorms?

[44] The second feature of Alfvén waves is that there are large, sharp decreases in the magnetic field magnitude at the phase-steepened edges of the waves. The decrease at $\sim 0410$ is very abrupt, while the ones at $\sim 0424$ and $\sim 0433$ UT are broader. These features have been called magnetic decreases (MDs) and magnetic holes (MHs) in interplanetary studies [Turner et al., 1977]. Similar features have been detected within the magnetosphere but have been called by different names. Studies have been performed to determine the plasma pressure within MDs and MHs. It was found that there is total pressure balance across the structures, i.e., the magnetic plus plasma thermal pressure is constant [Winterhalter et al., 1994b]. Discontinuities within propagating Alfvén waves have been simulated [Vasquez and Hollweg, 1996, 2001; Medvedev et al., 1997; Medvedev, 1999; Verkhoglyadova et al., 2003], but MDs and MHs have not. Tsubouchi and Matsumoto [2005] have produced MDs by one-dimensional hybrid simulations of rotational discontinuities interacting with the Earth's bow shock.

[45] What is the physical cause of these magnetic field decreases? The proton temperatures within large MDs have been examined in interplanetary space. Assuming bi-Maxwellian plasmas, the temperatures parallel and perpendicular to the magnetic field were determined. Temperatures were made both inside a MD and just outside the MD. The ratios $\mathrm{T}_{\perp \text { inside }} / \mathrm{T}_{\perp \text { outside }}$ and $\mathrm{T}_{\| \text {inside }} / \mathrm{T}_{\| \text {outside }}$ were calculated for each MD. Figure 16 shows the statistical result of the analysis of 32 MDs. For the parallel temperature ratio (bottom panel), the average value is close to 1.0 , with statistical fluctuations. Thus the parallel temperatures inside the MD are essentially the same as those outside. However, for the perpendicular temperature ratios (top panel), there is clear evidence that the temperatures inside the MDs are generally higher than those outside. This feature has been noted by Franz et al. [2000] and by Neugebauer et al. [2001]. It has been hypothesized that the physical mechanism is perpendicular (to the magnetic field) particle acceleration by the ponderomotive force associated with the phase-steepened edges of the waves [Tsurutani et al., 2002]. The perpendicularly heated ions create the MDs due to the diamagnetic effect from the particles. It should be mentioned that there are other possible mechanisms for MD 


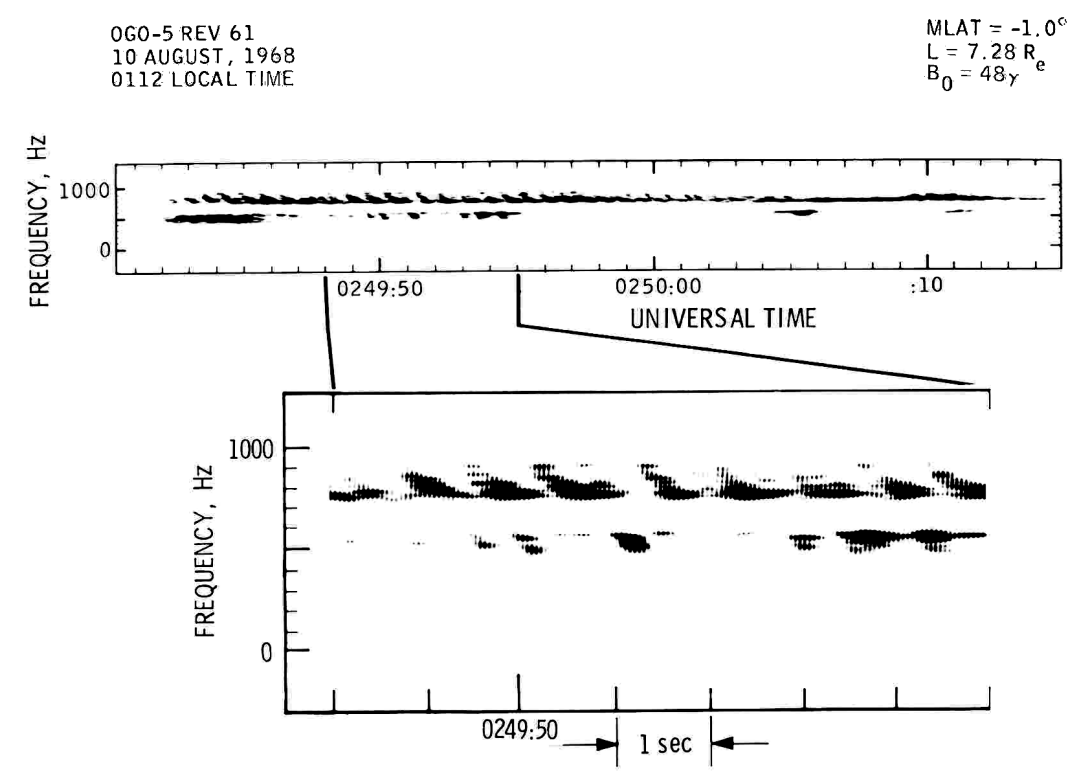

Figure 17. An example of the absorption of chorus amplitudes at frequencies near $\sim 0.5 \mathrm{f}_{\text {ce. }}$. The upper and lower frequency bands will cyclotron resonate with different energy electrons. It is believed that the lower band is more important for the parasitic energization of relativistic electrons. Taken from Tsurutani and Smith [1974].

formation: wave-wave interactions and other nonlinear Alfvén wave evolutionary features. The question of the exact mechanism of particle acceleration is still an open one. For a review of this topic, we refer the reader to Tsurutani et al. [2005].

[46] Magnetic field magnitude decreases within the MDs can be as large as $90 \%$ of the ambient magnetic field. If one assumes a plasma beta of $\sim 1.0$ at $1 \mathrm{AU}$ (the beta is the plasma thermal pressure $\left(\sum_{\mathrm{i}} \mathrm{n}_{\mathrm{i}} \mathrm{kT}\right.$ i $)$ divided by the magnetic pressure $\left.\left(\mathrm{B}^{2} / 8 \pi\right)\right)$, this implies that the beta within these structures can reach $\sim 200$. The plasma density increase is thus up to a factor $\sim 2.0$. Therefore when MDs impact the magnetosphere, there are significant ram pressure pulses of order 1.5 to 2.0 times the ambient pressures. It is possible that such impulses can be noted in magnetopause signatures as well as in dayside auroral intensifications.

\subsection{Relativistic Electron Acceleration During High-Speed Streams}

[47] Relativisitic electrons are detected within the magnetosphere/magnetotail during intervals of high-speed solar wind streams. The mechanism for acceleration is presently unknown, but the two classes of theories involve plasma waves. One mechanism entails electron radial diffusion within the magnetosphere due to long-period magnetic oscillations (PC5s) that break the particles third adiabatic invariant [Elkington et al., 1999; Hudson et al., 1999; Li et al., 2001; O’Brien et al., 2001; Mann et al., 2004; Miyoshi et al., 2004]. A second mechanism is energy diffusion by cyclotron resonant interactions with electromagnetic whistler mode waves called chorus [Summers et al., 1998, 2004; Horne and Thorne, 1998; Roth et al., 1999; Meredith et al., 2003]. This interaction breaks the particles' first adiabatic invariant.

[48] An example of electromagnetic chorus is shown in Figure 17. These emissions are highly structured in both frequency and time [Tsurutani and Smith, 1974, 1977; Nagano et al., 1996; Kasahara et al., 2001; Pickett et al., 2004]. These waves are generated by the loss cone instability [Kennel and Petschek, 1966] of substorm injected electrons in the equatorial region of the magnetosphere at $3<\mathrm{L}<9$ and in the local time region of $0<$ MLT $<13$ [Tsurutani and Smith, 1974; Meredith et al., 2001]. Kasahara et al. [2004] using Akebono data statistically studied chorus during 41 magnetic storms. They noted that chorus was located at $\mathrm{L} \sim 2.5$ during the beginning of the storm recovery phase and gradually moved back to $\mathrm{L} \sim 4$ in the late recovery phase. The frequency spacing between the chorus "elements" is $\sim 0.5 \mathrm{~s}$. The wave frequency range is $\sim 0.2$ to $0.7 \mathrm{f}_{\text {ce }}$, where $\mathrm{f}_{\text {ce }}$ is the equatorial electron cyclotron frequency. There is also a frequency "gap" at $\sim 0.5 \mathrm{f}_{\text {ce }}$ [Tsurutani and Smith, 1974]. It is believed that the frequency "gap" is due to Landau absorption [Tsurutani and Smith, 1974; Coroniti et al., 1984; Lauben et al., 2002; see also Horne et al., 2003]. The well-known $\sim 0.5 \mathrm{~s}$ temporal spacing between chorus elements or bursts has been addressed recently by Nunn et al. [1997] and Trakhtengerts et al. [2004]. It should be noted that the explanation for relativistic electron acceleration invokes chorus as important for a "parasitic" or secondary wave-particle interaction. Chorus is generated by the instability of 5-40 keV injected electrons in the processes discussed above.

[49] Figure 18 gives ACE solar wind parameters and geomagnetic activity data (Dst, $\mathrm{AU}, \mathrm{AL}$, and $\mathrm{AE}$ indices) for the interval of days 112 to 121,1998 . A high-speed stream is led by a fast forward shock at $\sim 1800$ UT day 113 , followed by a fast reverse shock at $\sim 2200$ UT. The plasma density (and ram pressure, P) therefore have a boxcar-like shape. Although a large substorm was apparently triggered by the fast forward shock, there was little or no Dst consequence at this time. The magnetic storm main phase was moderate, $D s t \sim-70 \mathrm{nT}$, and was associated with the 


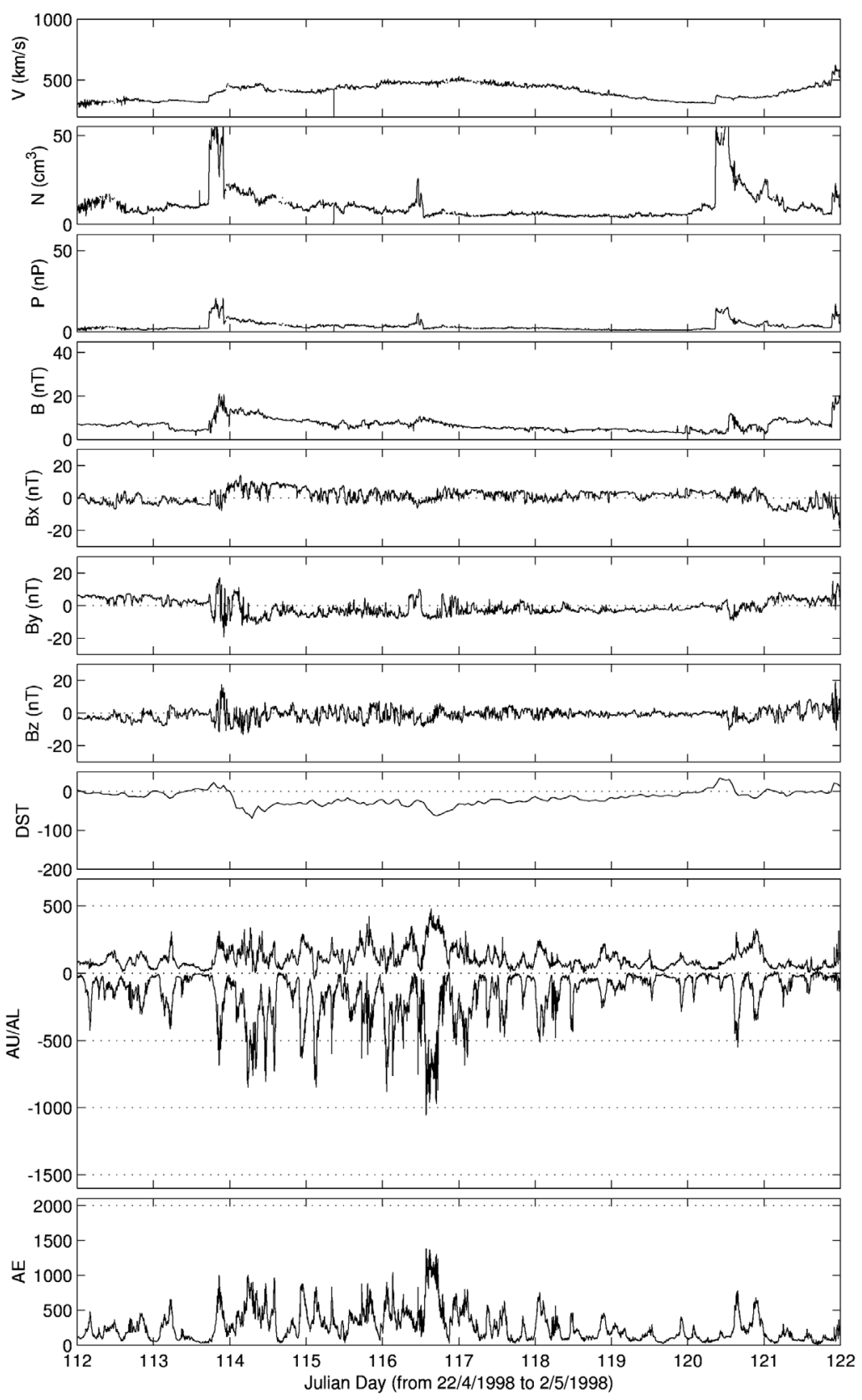

Figure 18. ACE solar wind parameters and resultant geomagnetic activity.

high-speed stream proper following the CIR. There was a long storm "recovery" phase extending from $\sim 0800$ UT day 114 until day 120. The interval between the start of the recovery phase and day 117 qualifies as a HILDCAA event. There are large-scale IMF $\mathrm{Bz}$ fluctuations during this interval (one cannot, however, expect to see correlations between the negative $\mathrm{Bz}$ intervals and $\mathrm{AE}$ increase intervals as shown earlier because the scale size of Alfvén waves are often smaller than the off-axis distance of the ACE spacecraft from the Sun-Earth line, a maximum value of $\sim 100 \mathrm{R}_{\mathrm{e}}$ (see discussion in the work of Crooker et al. [1982] and Baker et al. [1983])). Additionally, Alfvén waves propagate at $\sim 1 / 5$ to $1 / 10$ the solar wind convection speed, and therefore wave propagation effects will be important as well. The lack of interplanetary near-Earth orbiting spacecraft (such as IMP-8 as used in Figure 6) is a substantial loss for scientists trying to investigate details of the corotating stream space weather effects.

[50] Figure 19 shows the same interval but with different data panels than given in Figure 18. The top three panels are the Polar CAMICE $>230 \mathrm{keV}$ electron flux, the Akebono chorus amplitudes, and the Canadian chain of PC5 wave amplitudes. The chorus emissions were distinguished from plasmaspheric hiss [Smith et al., 1974] by analysis of Akebono spectrograms. The method of analysis is described by Kasahara et al. [2004]. Only waves in the local time 


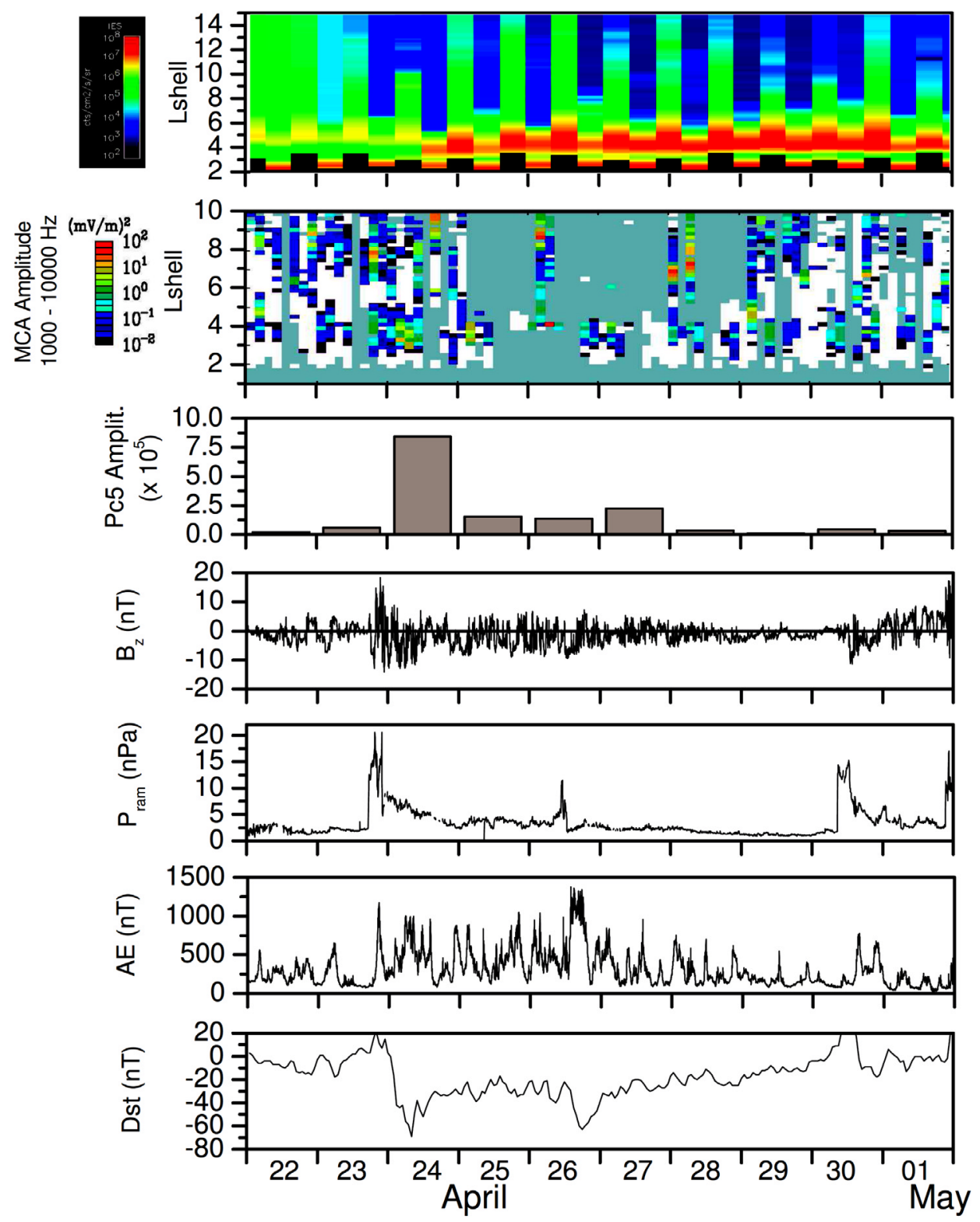

Figure 19. Relativistic electron fluxes (top panel), chorus electromagnetic emissions (second panel), and Pc5 amplitudes for the interval of Figure 17.

range of 0000-1300 MLT were examined. Although chorus is typically detected primarily close to the magnetic equator during substorms [Tsurutani and Smith, 1974], the sensitive wide band receiver and multichannel analyzers [Kimura et al., 1990] detected chorus during a magnetic storm in the off-equatorial regions as well [Miyoshi et al., 2003]. "Lack of data" regions are indicated by grey tones. The IMF Bz, solar wind ram pressure and $\mathrm{AE}$ and $D s t$ indices have been repeated for comparative purposes. Significant relativistic electron fluxes are noted in this small storm/high-speed stream event. We note that these $\mathrm{E}>230 \mathrm{keV}$ particles are not the highly relativistic $\mathrm{E}>1 \mathrm{MeV}$ reported in many works cited here, but these energetic particles represent an intermediate stage of energization. The relativistic electrons are noted from 25 through 30 April and beyond. As many previous researchers have noted, the electrons first appear in the "recovery" phase of the storm. The electrons appear at the beginning of 25 April, whereas the small storm begins at the start of 24 April. The largest amplitude PC5 waves are detected on the day of the storm, but significant amplitudes are still present on days 25 to 27 April as well. There is no particularly strong chorus emissions detected by Akebono, but there are some significant wave amplitudes from 24 to 28 April (note from the above discussion chorus during substorms are typically detected within $10^{\circ}$ to $15^{\circ}$ of the magnetic equator, especially in the local time range of 0000 to 0600 MLT). Chorus has a much broader latitudinal distribution from 0600 to 1300 MLT [Tsurutani and Smith, 1977]. Thus it is possible that "equatorial" chorus is missed during the magnetic storm when Akebono is in the midnight to dawn sector. This may be particularly true during the extended storm "recovery" phases when the plasma injections are weaker. Certainly, the maximum wave amplitudes 


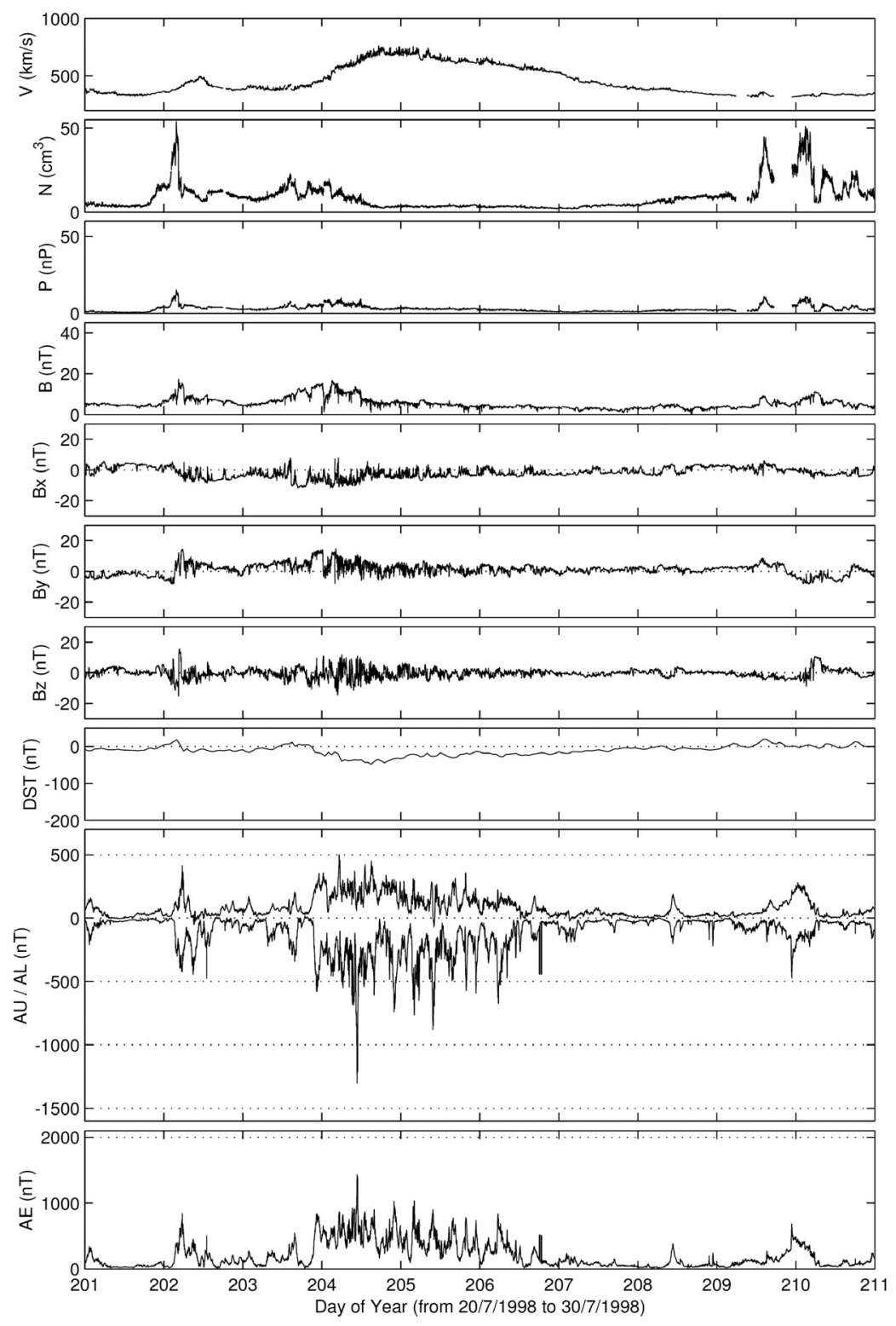

Figure 20. The format is the same as Figure 18, but for a very short duration solar wind stream.

which occur at the magnetic equator are not recorded in the figure.

[51] We show a second example of high-speed streams, Alfvén waves, magnetospheric waves, and relativistic electrons in Figures 20 and 21. The format is the same as for Figures 18 and 19. This event from days 204 to 207 was chosen because there was only a weak magnetic storm (Figure 20) that occurred. The peak (negative) Dst value was $\sim-42$ nT. There were interplanetary Alfvén waves and a HILDCAA event also present. The magnetic storm was on 23 July and the electrons first appeared on 24 July, in the magnetic storm "recovery" phase. The PC5 wave amplitudes were largest on 23 July and monotonically decreased through 25 July (see Posch et al. [2003] for a discussion of wave frequency bands during the storm initial, main, and recovery phases). In this event, the chorus emissions were quite intense, being highest on 23 July and less intense during 24 and 25 July. It is interesting to note that the electron flux levels remain high until 29 July. It is not known whether the electron loss process(es) are low during these latter days (26 to 29 July) or if continuous production (and loss) is still occurring. Unfortunately, the Akebono orbit is not ideal to determine whether chorus is continuously being generated in the deep "recovery" portion of the magnetic storm or not.

\section{Summary}

[52] Geomagnetic activity due to corotating solar wind streams has been reviewed. Corotating stream/CIR-induced magnetic storms have the same three phases: initial, main, and "recovery," as do ICME-induced magnetic storms. However, the interplanetary causes of each of the storm phases of corotating stream/CIR-induced magnetic storms are different from those of ICME-induced magnetic storms. The coroating stream-induced storm initial phases are 

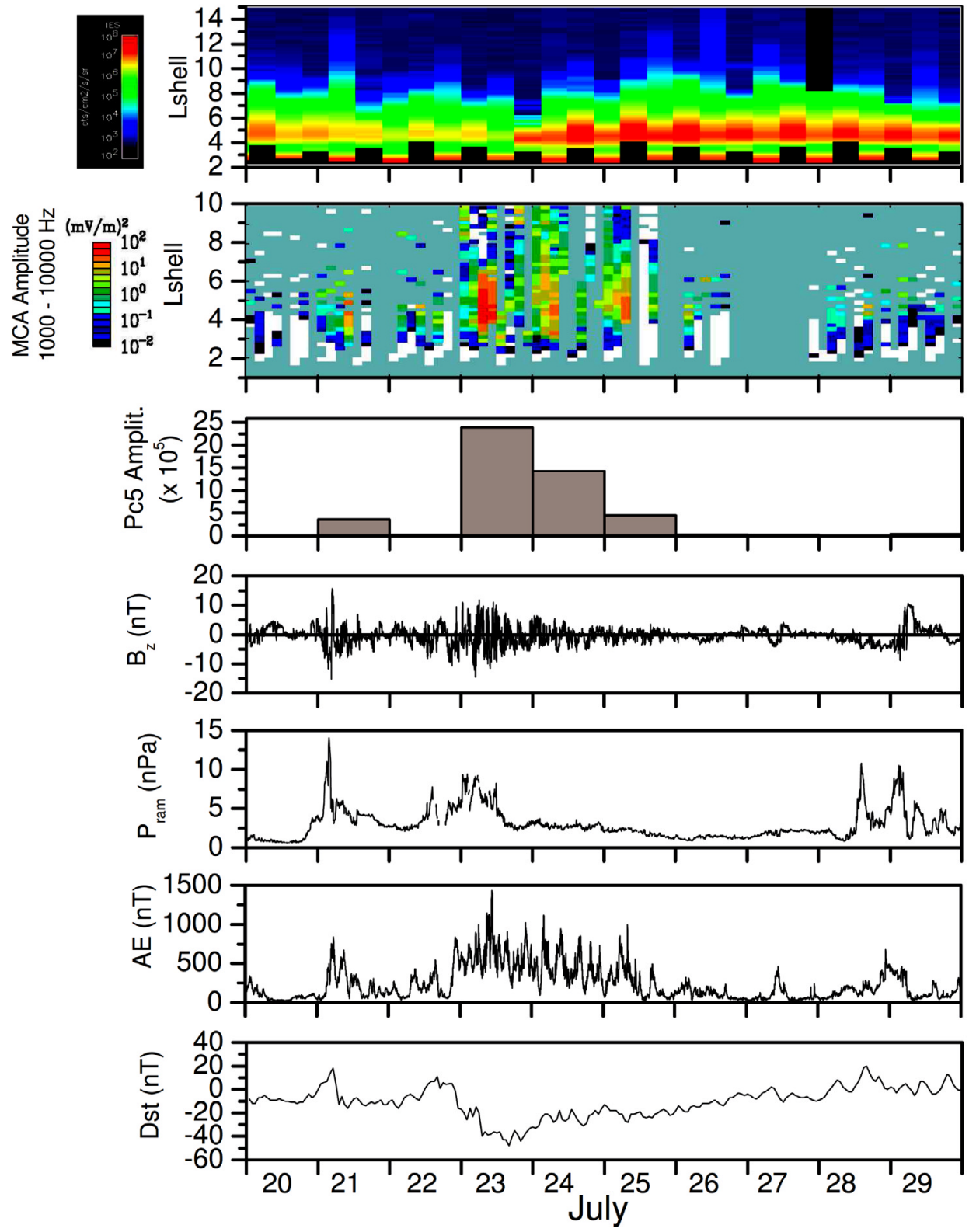

Figure 21. The format is the same as Figure 19, except for the interval of Figure 20.

caused by ram pressure increases from high plasma densities in the heliospheric current sheet plasma sheet (HCSPS) in the slow solar wind. The irregularly shaped, weak-tomoderate intensity storm main phases are caused by magnetic reconnection between the highly variable CIR southward Bz fluctuations and the Earth's magnetopause magnetic fields. High speed stream magnetic storm "recovery phases" are long-lasting and can have durations as long as a solar rotation ( $\sim 27$ days). High-speed stream storm "recovery" phases are not pure recoveries from an energy standpoint. Besides continuous loss of ring current particles by standard physical processes, fresh particles are also being continuously injected into the outer regions of the magnetosphere. One identified mechanism is magnetic reconnection between the southward component of interplanetary Alfvén waves and magnetopause fields. Other physical energy injection processes may be occurring as well.
[53] The appearance of long duration corotating solar wind streams at Earth is associated with the presence of solar coronal holes that either extend from the polar regions to near-equatorial regions or the appearance of isolated large near-equatorial coronal holes. The relative importance of the two has not been evaluated yet. The former type of coronal hole typically occurs in the declining phase of the solar cycle, well away from solar maximum. The number of CME releases are generally in phase with the solar cycle, resulting in the number of storms with $D s t<-100 \mathrm{nT}$ also being in phase (see Figure 12). Analyses of the temporal area of polar coronal holes over the solar cycle [Harvey et al., 2000; Harvey and Recely, 2002] provide a good idea of the geoeffectiveness of high speed streams over the solar cycle, but the effects of isolated equatorial coronal holes has yet to be evaluated. 
[54] Several observations discussed in this paper indicate that geomagnetic activity during the declining phase of the solar cycle is substantially different than during solar maximum. There are far fewer major $(D s t<-100 \mathrm{nT})$ magnetic storms, perhaps by factors of 10 to 20 (Figure 12). However, on the other hand, solar wind energy injection associated with the high speed stream Alfvén waves leads to long periods of high $\mathrm{AE} / \mathrm{AL}$ intervals. In the auroral regions, the auroras are continuous and extend over the entire auroral zone and higher latitudes. This high-latitude phenomena, averaged over a year or even shorter intervals is more energy intensive than during some major magnetic storms, at least from the point of view of electron precipitation into the ionosphere (see Guarnieri [2005] analyses over 4-day intervals). The impacts of this precipitation on ionospheric heating and thermal winds is a topic that needs to be studied.

[55] One puzzle is the nature of the auroras in the midnight sector and elsewhere. The general features of the auroras do not have the standard auroral substorm expansive phase signatures: brightening of arcs, then northward, westward, and eastward expansions. Instead, the auroras are broad, slowly-varying low-intensity displays covering all local times. Viewed by the Polar satellite from large ( 7.5 to $10 \mathrm{Re}$ ) distances above the poles, they appear as "diffuse" in nature (this is not the same definition as used by ground observers who have much higher spatial resolution). One might think these events as possible convection bays [Pytte et al., 1978; Sergeev et al., 1996], but there have been consistent observations that during these events, the $\mathrm{AL}$ index (westward electrojets) are much larger than the $\mathrm{AU}$ index (eastward electrojets), contrary to the signature of convection enhancements. These storm "recovery" phase events may indeed be a different and unique form of aurora and ionospheric/magnetospheric current system.

[56] Substorm expansion phases were shown to occur during HILDCAA events. However, the substorm current systems were masked by even more intense systems. An effort needs to be made to understand the global current system(s) during HILDCAA intervals. It is possible that contributions from local time sectors other than the midnight sector are playing an important role. The most potentially dangerous space weather effect of high-speed streams is the production of relativistic electrons. These could be damaging to orbiting spacecraft [Wrenn, 1995; Baker, 1996]. Arguments have been presented by others that the particles are not created by magnetic storm main phases but are produced by some mechanism or mechanisms in the extended "recovery" phases [Miyoshi et al., 2003]. Here it should be pointed out to the reader that these extended "recovery" phases are not limited to only coronal hole high-speed stream interactions, but are also found after ICME magnetic storm main phases as well (but for only 1 or 2 days). The high speed solar wind intervals have largeamplitude Alfvén waves, much like those in corotating solar wind streams. The southward component of the Alfvén waves present in these streams can produce HILDCAAs as well (F. L. Guarnieri et al., The nature of auroras during High-Intensity Long Duration Continuous AE (HILDCAA) events: 1998 to 2001, submitted to Brazilian Journal of Physics, 2006). This topic has been overshadowed by the often extremely intense magnetic storms caused by the fast
ICMEs. However, we argue that this topic merits study. Unanswered questions are: are the Alfvén wave properties similar to those in high-speed streams? Are the "recovery" phase AE/-AL increases indeed not substorm expansive phases? The only true test will be to examine imaging data.

[57] Relativistic electron acceleration has to date focused on either a radial diffusion mechanism or a chorus energy diffusion mechanism. The wave results presented here indicate that both PC5 waves and electromagnetic chorus are most intense during the storm initial and main phases and less intense in the extended "recovery" phases. Thus it would be expected that electron acceleration should follow accordingly. However for the $>230 \mathrm{keV}$ electrons shown here there is a general lack of electron enhancements during the storm main phases.

[58] Brautigam and Albert [2000], Obara et al. [2001], Meredith et al. [2002], and Posch et al. [2003] have suggested that an additional acceleration mechanism is need in the inner $(3<\mathrm{L}<5)$ region of the magnetosphere where radial diffusion is less effective. From Figures 19 and 21 it is clear that both waves are present at the same time, and it is probable that both mechanisms plus others (ram pressure pulses?) are operative. Future models should incorporate several mechanisms. Chorus energy diffusion should incorporate the possibility of phase-coherence of the emission elements and also oblique propagation of off-axis waves. The chorus intensity-local time distribution during HILDCAAs needs to be investigated. If the small injections of plasma sheet electrons is the source of chorus during these storm "recovery" phases, then the most likely location of intense emissions (with broad latitudinal extent) will be in the postdawn sector, as noted by previous studies during substorms. Models assuming chorus generation by left-over storm main phase electrons in an expanding plasmasphere could be ruled out.

[59] The southward components of Alfvén waves are believed to cause magnetic reconnection at the magnetopause and shallow injections of plasma sheet plasma on the nightside. It has been noted [Crooker et al., 1982; Baker et al., 1983] that interplanetary Alfvén waves can have wavelength scales that are smaller than the magnetosphere ( $\sim 30 \mathrm{Re})$. How does the magnetosphere react when reconnection is occurring on only part of the dayside magnetopause surface? What happens when MD pressure pulses impinge on only part of the magnetosphere?

[60] We hope the readers will find the paper interesting and informative. Perhaps more importantly, we hope that he/she will do research in some of the areas mentioned above.

\section{Appendix A: Glossary}

[61] Alfvén Wave (magnetohydrodynamic shear wave)-A transverse wave in magnetized plasma characterized by a change of direction of the magnetic field with no change in either the magnitude of the field or the plasma density. This description gives the basic concepts of small-amplitude MHD Alfvén waves. The waves discussed in this paper are "kinetic" and nonlinear.

[62] CIR- Corotating Interaction Region, created by the interaction/collision of a high-speed stream with a slowspeed stream. 
[63] Convection Bays- Auroral zone geomagnetic activity that have a DP2 current system [Nishida, 1968a, 1968b], representing an enhancement of magnetospheric convection. There are no auroral breakups and no substorm electrojets. Convection bays are characterized by large eastward electrojets on the dusk side that are larger than or equal to the westward electrojet at dawn ( $\mathrm{AU}>-\mathrm{AL})$. They can reach intensities of $\mathrm{AE}=500$ to $1000 \mathrm{nT}$.

[64] CME- Coronal Mass Ejection, a transient outflow of plasma from or through the solar corona.

[65] Coronal Holes- Extended regions of the solar corona characterized by exceptionally low densities and unipolar photospheric magnetic fields having "open" magnetic field topologies. Coronal holes are largest and most stable at or near the solar poles and are a source of high-speed (750$800 \mathrm{~km} / \mathrm{s}$ ) solar wind. Coronal holes are visible in several wavelengths, most notably solar $\mathrm{X}$ rays visible only from space but also in the He $1083 \mathrm{~nm}$ line which is detectable from the surface of the Earth. In soft X-ray images (photon energy of $\sim 0.1-1.0 \mathrm{keV}$ or a wavelength of $10-100 \AA$ ), these regions are dark, thus the name "holes."

[66] GSM- Geomagnetic Solar Magnetospheric, a coordinate system used for magnetospheric measurements.

[67] HCS- Heliospheric Current Sheet, a surface dividing the northern and southern magnetic field hemispheres in the solar wind.

[68] HCSPS- Heliospheric Current Sheet Plasma Sheet, a high-density plasma region surrounding the HCS.

[69] HILDCAA- High Intensity, Long-Duration, Continuous AE Activity.

[70] ICME- Interplanetary Coronal Mass Ejection, the interplanetary remains/evolution of a CME.

[71] Magnetic Storm-A worldwide disturbance of the Earth's magnetic field, distinct from regular diurnal variations.

[72] Initial Phase: That interval when there may be an increase of the near-equatorial and middle-latitude horizontal magnetic field intensity at the surface of the Earth. The initial phase can last for hours (up to a day), but some storms proceed directly into the main phase without showing an initial phase.

[73] Main Phase: Of a magnetic storm, that interval when the horizontal magnetic field at near-equatorial and middle latitudes decreases, owing to the effects of an increasing magnetospheric ring current.

[74] Recovery Phase: Of a magnetic storm, that interval when the depressed horizontal fields return to normal levels.

[75] Shock Wave (collisionless)-A shock wave is characterized by a discontinuous change in pressure, density, temperature, and particle streaming velocity, propagating through a compressible fluid or plasma. Collisionless shock waves occur in the solar wind when the fast solar wind overtakes slow solar wind, with the difference in speeds being greater than the magnetosonic speed.

[76] Solar Cycle- The approximately 22 year solar sunspot number cycle. Each half cycle of $\sim 11$ years is asymmetric, rising to a peak in 3 to 5 years and declining over 6 to 8 years. Pertinent to this paper, fast CME releases maximize just prior to and just after solar maximum (see Figure 12). Harvey et al. [2000] and Harvey and Recely [2002] have shown that polar coronal holes are very small to nonexistent at solar maximum. Shortly after new polar coronal holes form, they rapidly expand. Their expansion is not monotonic. They reach maximum area around solar minimum, after which there is a rapid decrease in area as the new cycle activity builds.

[77] Declining Phase- The portion of the solar cycle between solar maximum and solar minimum.

[78] Solar Maximum- The month(s) during the sunspot cycle when the smoothed sunspot number reaches a maximum.

[79] Solar Minimum-The month(s) during the sunspot cycle when the smoothed sunspot number reaches a minimum.

[80] SSC- Storm Sudden Commencement, a SI which is followed by a magnetic storm.

[81] SI- Sudden Impulse, an abrupt increase in the magnetic field at the surface of the Earth; this is caused by a significant ram pressure increase onto the magnetosphere.

[82] For further acronyms and definitions, we refer the reader to Suess and Tsurutani [1998].

[83] Acknowledgments. Portions of this research were performed at the Jet Propulsion Laboratory, California Institute of Technology under contract with NASA and portions were performed at RISH, Kyoto University, Uji Japan. We thank the participants of the Henningsvaer, Norway 2003 workshop and the Chapman 2005 Manaus Conference for opportunities to present this material and gain valuable feedback. We thank J. Harvey (and K. Harvey) for extended discussions over the years on the topic of the evolution of coronal holes. We are indebted to G.S. Lakhina and O.P. Verkhoglyadova for critical reading of the typescript. BTT wishes to thank T. Tsuda for his generous hospitality while visiting at RISH. This represents the fourth in a series of review articles on different aspects of magnetic storms [Gonzalez et al., 1994; Kamide et al., 1998; Daglis et al., 2003].

[84] Shadia Rifai Habbal thanks Richard B. Horne and Bernard J. Vasquez for their assistance in evaluating this paper.

\section{References}

Akasofu, S.-I. (1964), The development of the auroral substorm, Planet. Space Sci., 12, 273

Akasofu, S.-I. (1981), Energy coupling between the solar wind and the magnetosphere, Space Sci. Rev., 28, 121.

Baker, D. N. (1996), Soar wind-magnetosphere drivers of space weather, J. Atmos. Terr. Phys, 58, 1509.

Baker, D. N., R. D. Zwickl, S. J. Bame, E. W. Hones Jr., B. T. Tsurutani, E. J. Smith, and S.-I. Akasofu (1983), An ISEE high time resolution study of interplanetary parameter correlations with magnetospheric activity, J. Geophys. Res., 88, 6230.

Baker, D. N., J. B. Blake, R. W. Klebesadel, and P. R. Higbie (1986), Highly relativistic electrons in the Earth's outer magnetosphere: 1. Lifetimes and temporal history 1979-1984, J. Geophys. Res., 91, 4265.

Baker, D. N., S. G. Kanekal, T. I. Pulkkinen, and J. B. Blake (2001), Equinoctial and solstitial averages of magnetospheric relativistic electrons: A strong semiannual modulation, Geophys. Res. Lett., 106, 19,169. Balogh, A., E. J. Smith, B. T. Tsurutani, D. J. Southwood, R. J. Forsyth, and T. S. Horbury (1995), The heliospheric magnetic field over the south polar region of the sun, Science, 268, 1007.

Balogh, A., J. T. Gosling, J. R. Jokipii, R. Kallenbach, and H. Kunow (Eds.) (1999), Corotating interaction regions, Space Sci. Rev., 89, 141 411.

Belcher, J. W., and L. Davis Jr. (1971), Large-amplitude Alfvén waves in the interplanetary medium: 2, J. Geophys. Res., 76, 3534.

Brautigam, D. H., and J. M. Albert (2000), Radial diffusion analysis of outer radiation belt electrons during the October 9, 1990 magnetic storm, J. Geophys. Res., 105, 291.

Burlaga, L. F. (1969), Directional discontinuities in the interplanetary magnetic field, Sol. Phys., 7, 54.

Cohen, R. H., and R. M. Kulsrud (1974), Nonlinear evolution of parallel propagating hydromagnetic waves, Phys. Fluids, 17, 2215.

Cornwall, J. M., F. V. Coroniti, and R. M. Thorne (1970), Turbulent loss of ring current protons, J. Geophys. Res., 75, 4699.

Cornwall, J. M., F. V. Coroniti, and R. M. Thorne (1971), Unified theory of SAR arc formation at the plasmapause, J. Geophys. Res., 76, 4428.

Coroniti, F. V., F. L. Scarf, C. F. Kennel, and W. S. Kurth (1984), Analysis of chorus emission at Jupiter, J. Geophys. Res., 89, 3801. 
Crooker, N. U., G. L. Siscoe, C. T. Russell, and E. J. Smith (1982), Factors controlling degree of correlation between ISEE 1 and ISEE 3 interplanetary magnetic field measurements, J. Geophys. Res., 87, 2224.

Daglis, I. A., J. U. Kozyra, Y. Kamide, D. Vassiliadis, A. S. Sharma, M. W. Liemohn, W. D. Gonzalez, B. T. Tsurutani, and G. Lu (2003), Intense space storms: Critical issues and open disputes, J. Geophys. Res., 108(A5), 1208, doi:10.1029/2002JA009722.

Elkington, S. R., M. K. Hudson, and A. A. Chan (1999), Acceleration of relativistic electrons via drift-resonant interaction with toroidal-mode Pc-5 ULF oscillations, Geophys. Res. Lett., 26, 3273.

Elkington, S. R., M. K. Hudson, and A. A. Chan (2003), Resonant acceleration and diffusion of outer zone electrons in an asymmetric geomagnetic field, J. Geophys. Res., 108(A3), 1116, doi:10.1029/2001JA009202.

Farrugia, C. J., L. F. Burlaga, and R. P. Lepping (1997), Magnetic clouds and their quiet-storm effects at Earth, in Magnetic Storms, Geophys. Monogr. Ser., vol. 98, edited by B. T. Tsurutani et al., p. 91, AGU, Washington, D. C

Feldstein, Y. I., et al. (2005), Self-consistent modeling of the large-scale distortions in the geomagnetic field during the 24-27 September 1998 major magnetic storm, J. Geophys. Res., 110, A11214, doi:10.1029/ 2004JA010584.

Franz, M., et al. (2000), Magnetic field depressions in the solar wind, J. Geophys. Res., 105, 12,725.

Gonzalez, A. L. C., and W. D. Gonzalez (1987), Periodicities in the interplanetary magnetic field polarity, J. Geophys. Res., 92, 4357

Gonzalez, W. D., A. L. Clua de Gonzalez, and B. T. Tsurutani (1990), Dualpeak solar cycle distribution of intense geomagnetic storms, Planet. Space Sci., 38, 181.

Gonzalez, W. D., J. A. Joselyn, Y. Kamide, H. W. Kroehl, G. Rostoker, B. T. Tsurutani, and V. M. Vasyliunas (1994), What is a geomagnetic storm?, J. Geophys. Res., 99, 5771.

Guarnieri, F. L. (2005), A study of the interplanetary and solar origin of high intensity long duration and continuous auroral activity events, Ph.D. thesis, Inst. Nac. Pesqui. Espaciais, Sao Jose dos Campos, Brazil.

Harvey, K. L., and F. Recely (2002), Polar coronal holes during cycles 22 and 23, Sol. Phys., 211, 31

Harvey, K., S. Suess, M. Aschwanden, M. Guhathakurta, J. Harvey, D. Hathaway, B. LaBonte, N. Sheeley, and B. T. Tsurutani (2000), NASA white paper derived from Workshop on Coronal Holes Near Solar Maximum and Over the Solar Cycle, NASA, Washington, D. C.

Hollweg, J. V., and P. A. Isenberg (2002), Generation of the fast wind: A review with emphasis on the resonant cyclotron interaction, J. Geophys. Res., 107(A7), 1147, doi:10.1029/2001JA000270.

Horne, R. B. (2002), The contribution of wave-particle interactions to electron loss and acceleration in the Earth's radiation belts during geomagnetic storms, in Review of Radio Science 1999-2002, edited by W. R. Stone, p. 801, John Wiley, Hoboken, N. J.

Horne, R. B., and R. M. Thorne (1998), Potential wave modes for electron scattering and stochastic acceleration to relativistic energies during magnetic storms, Geophys. Res. Lett., 25, 3011

Horne, R. B., N. P. Meredith, R. M. Thorne, D. Heynderickx, R. H. A. Iles, and R. R. Anderson (2003), Evolution of energetic electron pitch angle distributions during storm time electron acceleration to $\mathrm{MeV}$ energies, J. Geophys. Res., 108(A1), 1017, doi:10.1029/2002JA009468

Hudson, M. K., S. R. Elkington, J. G. Lyon, C. C. Goodrich, and T. J. Rosenberg (1999), Simulations of radiation belt dynamics driven by solar wind variations, in Sun-Earth Plasma Connections, edited by J. Burch, R. L. Carovillano, and S. K. Antiochos, p. 171, AGU, Washington, D. C.

Iyemori, T. (1980), Time delay of the substorm onset from the IMF southward turning, J. Geomagn. Geoelectr., 32, 267.

Kamide, Y., et al. (1998), Current understanding of magnetic storms: Storm-substorm relationships, J. Geophys. Res., 103, 17,705.

Kasahara, Y., I. Nagano, and I. Kimura (2001), Global dynamics of the inner magnetosphere derived from ELF/VLF waves observed by Akebono, paper presented at 2001 Asia-Pacific Radio Science Conference, Chuo Univ., Tokyo, Japan.

Kasahara, Y., H. Uchiyama, and Y. Goto (2004), Whistler mode chorus observed around the plasmapause during magnetic storm, in Frontiers in Magnetic Plasma Physics, COSPAR Colloq. Ser., vol. 16, edited by M. Hoshino, Y. Omura, and L. J. Lanzerotti, p. 228, Elsevier, New York.

Kennel, C. F., and H. E. Petschek (1966), Limit on stably trapped particle fluxes, J. Geophys. Res., 71, 1.

Kimura, I., K. Hashimoto, I. Nagano, T. Okada, M. Yamamoto, T. Yoshino, H. Matsumoto, M. Ejiri, and K. Hayashi (1990), VLF observations by the Akebono (EXOS-D) satellite, J. Geomagn. Geoelectr., 42, 459.

Klein, L. W., and L. F. Burlaga (1982), Interplanetary magnetic clouds at 1 AU, J. Geophys. Res., 87, 613

Kozyra, J. U., V. K. Jordanova, R. B. Horne, and R. M. Thorne (1997) Modeling of the contribution of electromagnetic ion cyclotron (EMIC) waves to stormtime ring current erosion, in Magnetic Storms, Geophys.
Monogr. Ser., vol. 98, edited by B. T. Tsurutani et al., pp. 187, AGU, Washington, D. C.

Kozyra, J. U., M. W. Liemohn, C. R. Clauer, A. J. Ridley, M. F. Thomsen, J. E. Borovsky, J. L. Raeder, V. K. Jordanova, and W. D. Gonzalez (2002), Multistep Dst development and ring current composition changes during the 4-6 June 1991 magnetic storm, J. Geophys. Res., 107(A8), 1224, doi:10.1029/2001JA000023.

Krieger, A. S., A. F. Timothy, and E. C. Roelof (1973), A coronal hole and its identification as the source of a high velocity solar wind stream, Sol Phys., 23, 123.

Lauben, D. S., U. S. Inan, T. F. Bell, and D. A. Gurnett (2002), J. Geophys Res., 107(A12), 1429, doi:10.1029/2000JA003019.

Lepping, R. P., J. A. Jones, and L. F. Burlaga (1990), Magnetic field structure of interplanetary magnetic clouds at $1 \mathrm{AU}, J$. Geophys. Res., 95, 11,957.

Lepping, R. P., D. B. Berdichevsky, C. C. Wu, A. Szabo, T. Narock, F. Mariani, A. J. Lazarus, and A. J. Quivers (2006), A summary of WIND magnetic clouds for the years 1995-2003: Model-fitted parameters, associated errors, and classifications, Ann. Geophys., 24, 215.

Li, X., and M. A. Temerin (2001), The electron radiation belt, Space Sci. Rev., 95, 569.

Li, X., M. Temerin, D. N. Baker, G. D. Reeves, and D. Larson (2001), Quantitative prediction of radiation belt electrons at geostationary orbit based on solar wind measurements, Geophys. Res., Lett., 28, 1887.

Lyons, L. R., G. T. Blanchard, J. C. Sampson, R. P. Lepping, T. Yamamoto, and T. Moretto (1997), Coordinated observations demonstrating external substorm triggering, J. Geophys. Res., 102, 27,039.

Mann, I. R., T. P. O'Brien, and D. K. Milling (2004), Correlations between ULF wave power, solar wind speed, and relativistic electron flux in the magnetosphere: Solar cycle dependence, J. Atmos. Sol. Terr. Phys., 66 , 187.

McComas, D. J., et al. (2002), Ulysses second fast latitude scan; complexity near solar maximum and the reformation of polar coronal holes, Geophys. Res. Lett., 29(9), 1290, doi:10.1029/2001GL014164.

Medvedev, M. V. (1999), Collisionless dissipative nonlinear Alfven waves: Nonlinear steepening, compressible turbulence, and particle trapping, Phys. Plasmas, 6(5), 2191

Medvedev, M. V., V. I. Shevchenko, P. H. Diamond, and V. L. Galinsky (1997), Fluid models for kinetic effects on coherent nonlinear Alfvén waves. II. Numerical solutions, Phys. Plasmas, 4(5), 1257.

Meredith, N. P., R. B. Horne, and R. R. Anderson (2001), Substorm dependence of chorus amplitudes: Implications for the acceleration of electrons to relativistic energies, J. Geophys. Res., 106, 13,165.

Meredith, N. P., R. B. Horne, D. Summers, R. M. Thorne, R. H. A. Iles, D. Heynderickx, and R. R. Anderson (2002), Evidence for acceleration of outer zone electrons to relativistic energies by whistler-mode chorus, Ann. Geophys., 20, 967.

Meredith, N. P., M. Cain, R. B. Horne, R. M. Thorne, D. Summers, and R. R. Anderson (2003), Evidence for chorus-driven electron acceleration to relativistic energies from a survey of geomagnetically disturbed periods, J. Geophys. Res., 108(A6), 1248, doi:10.1029/2002JA009764.

Miyoshi, Y., A. Morioka, T. Obara, H. Misawa, T. Nagai, and Y. Kasahara (2003), Rebuilding process of the outer radiation belt during the 3 November 1993 magnetic storm: NOAA and Exos-D observations, J. Geophys. Res., 108(A1), 1004, doi:10.1029/2001JA007542.

Miyoshi, Y., V. K. Jordanova, A. Morioka, and D. S. Evans (2004), Solar cycle variations of the electron radiation belts: Observations and radial diffusion simulation, Space Weather, 2, S10S02, doi:10.1029/ 2004SW000070.

Nagano, I., S. Yagitani, H. Kojima, and H. Matsumoto (1996), Analysis of wave normal and Poynting vectors of the chorus emissions observed on Geotail, J. Geomagn. Geoelectr., 48, 299.

Nerney, S., and S. T. Suess (2005), Stagnation flow in the thin streamer boundaries, Astrophys. J., 624, 378.

Neugebauer, M., et al. (2001), Ion distributions in large magnetic holes in the fast solar wind, J. Geophys. Res., 106, 5635.

Neupert, W. M., and V. Pizzo (1974), Solar coronal holes as sources of recurrent geomagnetic disturbances, J. Geophys. Res., 79, 3701.

Nishida, A. (1968a), Geomagnetic DP2 fluctuations and associated magnetospheric phenomena, J. Geophys. Res., 73, 1795.

Nishida, A. (1968b), Coherence of geomagnetic DP 2 fluctuations with interplanetary magnetic variations, J. Geophys. Res., 73, 5549.

Nunn, D., Y. Omura, H. Matsumoto, I. Nagano, and S. Yagitani (1997), The numerical simulation of VLF chorus and discrete emissions observed on the Geotail satellite using a Vlasov code, J. Geophys. Res., 102, 27,083.

Obara, T., Y. Miyoshi, and A. Morioka (2001), Large enhancement of the outer belt during magnetic storms, Earth Planets Space, 53, 1163.

O'Brien, T. P., R. L. McPherron, D. Sornette, G. D. Reeves, R. Friedel, and H. J. Singer (2001), Which magnetic storms produce relativistic electrons at geosynchronous orbit?, J. Geophys. Res., 106, 15,533. 
Oieroset, M., J. Raeder, T. D. Phan, S. Wing, J. P. McFadden, W. Li, M. Fujimoto, H. Reme, and A. Balogh (1988), Global cooling and densification of the plasmasheet during an extended period of purely northward IMF on October 22-24, 2003, Geophys. Res. Lett., 32, L12S07, doi:10.1029/2004GL021523.

Parker, E. N. (1988), Nanoflares and the solar x-ray corona, Astrophys. J., $330,474$.

Paulikas, G., and J. B. Blake (1979), Effects of the solar wind on magnetospheric dynamics: Energetic electrons at the synchronous orbit, in Quantitative Modeling of Magnetospheric Processes, Geophys. Monogr. Ser. vol. 21, edited by W. Olsen, p. 21, AGU, Washington, D. C.

Phillips, J. L., S. J. Bame, W. C. Feldman, B. E. Goldstein, J. T. Gosling, C. M. Hammond, D. J. McComas, M. Neugebauer, E. E. Scime, and S. T. Suess (1995), Ulysses solar wind plasma observations at high southerly latitudes, Science, 268, 1030

Pickett, J. S., et al. (2004), Multi-point Cluster observations of VLF risers, fallers and hooks at and near the plasmapause, in Multiscale Processes in the Earth's Magnetosphere: From Interball to Cluster, edited by J. A. Sauvaud and Z. Nemecek, p. 307, Springer, New York.

Pizzo, V. J. (1985), Interplanetary shocks on the large scale: A retrospective on the last decade's theoretical efforts, in Collisionless Shocks in the Heliosphere: Reviews of Current Research, Geophys. Monogr. Ser., vol 35, edited by B. T. Tsurutani and R. G. Stone, p. 51, AGU, Washington, D. C.

Posch, J. L., M. J. Engebretson, V. A. Pilipenko, W. J. Hughes, C. T. Russell, and L. J. Lanzerotti (2003), Characterizing the long-period ULF response to magnetic storms, J. Geophys. Res., 108(A1), 1029 doi:10.1029/2002JA009386.

Pulkkinen, T. I., N. Yu. Ganushkina, E. I. Kallio, G. Lu, D. N. Baker, N. E. Turner, T. A. Fritz, J. F. Fennell, and J. Roeder (2002), Energy dissipation during a geomagnetic storm: May 1998, Adv. Space Res. $30,2231$.

Pytte, T., R. L. McPherron, E. W. Hones Jr., and H. I. West (1978), Multiple satellite studies of magnetospheric substorms: Distinction between polar magnetic substorm and convection driven negative bay, Planet. Space Sci., 83, 663 .

Roth, I., M. A. Temerin, and M. K. Hudson (1999), Resonant enhancement of relativistic electron fluxes during geomagnetically active periods, Ann. Geophys., 17, 631.

Sandanger, M. I., F. Soraas, K. Aarsnes, K. Oksavik, D. S. Evans, and M. S Greer (2005), Proton injections into the ring current associated with $\mathrm{Bz}$ variations during HILDCAA events, in The Inner Magnetosphere: Phy sics and Modeling, Geophys. Monogr. Ser, vol. 155, edited by T. I Pulkkinen, N. A. Tsygenenko, and R. H. W. Friedel, p. 2249, AGU, Washington, D. C

Sergeev, V. A., R. J. Pellinen, and T. I. Pulkkinen (1996), Steady magnetospheric convection: A review of recent results, Space Sci. Rev., 75, 551.

Sheeley, N. R., Jr., J. W. Harvey, and W. C. Feldman (1976), Coronal holes, solar wind streams and recurrent geomagnetic disturbances: 1973-1976, Solar Phys., 49, 271.

Sheeley, N. R., Jr., J. R. Asbridge, S. J. Bame, and J. W. Harvey (1977), A pictoral comparison of interplanetary magnetic field polarity, solar wind speed, and geomagnetic disturbance index during the sunspot cycle, Sol. Phys, 52, 485 .

Smith, E. J., and J. H. Wolfe (1976), Observations of interaction regions and corotating shocks between one and five AU: Pioneers 10 and 11, Geophys. Res. Lett., 3, 137.

Smith, E. J., A. M. A. Frandsen, B. T. Tsurutani, R. M. Thorne, and K. W. Chan (1974), Plasmaspheric hiss intensity variations during magnetic storms, J. Geophys. Res., 79, 2507.

Soraas, F., K. Aarsnes, K. Oksavik, M. I. Sandanger, D. S. Evans, and M. S. Greer (2004), Evidence for particle injection as the cause of Dst reduction during HILDCAA events, J. Atmos. Sol. Terr. Phys., 66, 177.

Soraas, F., K. Aarsnes, D. V. Carlsen, K. Oksavik, and D. S. Evans (2005), Ring current behavior as revealed by energetic proton precipitation, in The Inner Magnetosphere: Physics and Modeling, Geophys. Monogr. Ser., vol. 155, edited by T. I. Pulkkinen, N. A. Tsygenenko, and R. H. W. Friedel, p. 237, AGU, Washington, D. C.

Suess, S. T., and S. F. Nerney (2002), Stagnation flow in streamer boundaries, Astrophys. J., 565, 1275.

Suess, S. T., and B. T. Tsurutani (Eds.) (1998), From the Sun: Auroras, Magnetic Storms, Solar Flares, Cosmic Rays, Spec. Publ., vol. 50, AGU, Washington, D. C.

Summers, D., R. M. Thorne, and F. Xiao (1998), Relativistic theory of wave-particle resonant diffusion with application to electron acceleration in the magnetosphere, J. Geophys. Res., 103, 20,487.

Summers, D., C. Ma, N. P. Meridith, R. B. Horne, R. M. Thorne, and R. R. Anderson (2004), Modeling outer-zone relativistic electron response to whistler mode chorus activity during substorms, J. Atmos. Sol. Terr. Phys., 66, 133.
Thorne, R. M., and C. F. Kennel (1971), Relativistic electron precipitation during magnetic storm main phase, J. Geophys. Res., 76, 4446.

Trakhtengerts, V. Y., M. J. Rycroft, D. Nunn, and A. G. Demekhov (2003), Cyclotron acceleration of radiation belt electrons by whistlers, J. Geophys. Res., 108(A3), 1138, doi:10.1029/2002JA009559.

Trakhtengerts, V. Y., A. G. Demekhov, E. E. Titova, B. V. Kozelov, O. Santolik, D. Gurnett, and M. Parrot (2004), Interpretation of Cluster data on chorus emissions using the backward wave oscillator model, Phys. Plasmas, 11, 1345.

Tsubouchi, K, and H. Matsumoto (2005), Effect of upstream rotational field on the formation of a magnetic depression in a quasi-perpendicular shock downstream, J. Geophys. Res., 110, A04101, doi:10.1029/ 2004JA010818.

Tsurutani, B. T. (2000), Solar/interplanetary plasma phenomena causing geomagnetic activity at Earth, in Proceedings of International School of Physics "Enrico Fermi" Course CXLII, edited by B. Coppi, A. Ferrari, and E. Sindoni, p. 273, IOS Press, Amsterdam.

Tsurutani, B. T., and W. D. Gonzalez (1987), The cause of high intensity long-duration continuous AE activity (HILDCAAs): Interplanetary Alfvén wave trains, Planet. Space Sci., 35, 405

Tsurutani, B. T., and W. D. Gonzalez (1995), The efficiency of "viscous interaction" between the solar wind and the magnetosphere during intense northward IMF events, Geophys. Res. Lett., 22, 663.

Tsurutani, B. T., and W. D. Gonzalez (1997), The interplanetary causes of magnetic storms: A review, in Magnetic Storms, Geophys. Monogr. Ser. vol. 98, edited by B. T. Tsurutani et al., p. 77, AGU, Washington, D. C.

Tsurutani, B. T., and E. J. Smith (1974), Postmidnight chorus: A substorm phenomenon, J. Geophys. Res., 79, 118.

Tsurutani, B. T., and E. J. Smith (1977), Two types of magnetospheric ELF chorus and their substorm dependences, J. Geophys. Res., 82, 5112.

Tsurutani, B. T., E. J. Smith, W. D. Gonzalez, F. Tang, and S.-I. Akasofu (1988), Origin of interplanetary southward magnetic fields responsible for major magnetic storms near solar maximum (1978-1979), J. Geophys. Res., 93, 8519.

Tsurutani, B. T., C. M. Ho, E. J. Smith, M. Neugebauer, B. E. Goldstein, J. S. Mok, J. K. Arballo, A. Balogh, D. J. Southwood, and W. C. Feldman (1994), The relationship between interplanetary discontinuities and Alfvén waves: Ulysses observations, Geophys. Res. Lett., 21, 2267.

Tsurutani, B. T., W. D. Gonzalez, A. L. C. Gonzalez, F. Tang, J. K. Arballo, and M. Okada (1995), Interplanetary origin of geomagnetic activity in the declining phase of the solar cycle, J. Geophys. Res., 100, 21,717.

Tsurutani, B. T., K.-H. Glassmeier, and F. Neubauer (1997), A review of nonlinear low frequency (LF) wave observations in space plasmas: On the development of plasma turbulence, in Nonlinear Waves and Chaos in Space Plasmas, edited by T. Hada and H. Matsumoto, p. 1, Terra Sci., Tokyo.

Tsurutani, B. T., B. Dasgupta, C. Galvan, M. Neugebauer, G. S. Lakhina, J. K. Arballo, D. Winterhalter, B. E. Goldstein, and B. Buti (2002), Phasesteepened Alfvén waves, proton perpendicular energization and the creation of magnetic holes and magnetic decreases: The ponderomotive force, Geophys. Res. Lett., 29(24), 2233, doi:10.1029/2002GL015652.

Tsurutani, B. T., W. D. Gonzalez, F. Guarnieri, Y. Kamide, X. Zhou, and J. K. Arballo (2004), Are high-intensity long-duration continuous AE activity (HILDCAA) events substorm expansion events?, J. Atmos. Sol. Terr. Phys., 66, 167.

Tsurutani, B. T., G. S. Lakhina, J. S. Pickett, F. L. Guarnieri, N. Lin, and B. E. Goldstein (2005), Nonlinear Alfvén waves, discontinuities, proton perpendicular acceleration, and magnetic holes/decreases in interplanetary space and the magnetosphere: Intermediate shocks?, Nonlinear Proc. Geophys., 12, 321

Turner, J. M., L. F. Burlaga, N. F. Ness, and J. F. Lemaire (1977), Magnetic holes in the solar wind, J. Geophys. Res., 82, 1921.

Turner, N. E., D. N. Baker, T. I. Pulkkinen, and R. L. McPherron (2000), Evaluation of the tail current contribution to Dst, J. Geophys. Res., 105, 5431.

Vasquez, B. J., and J. V. Hollweg (1996), Formation of arc-shaped Alfvén waves and rotational discontinuities from oblique linearly polarized wave trains, J. Geophys. Res., 101, 13,527.

Vasquez, B. J., and J. V. Hollweg (2001), Evolution and dissipation of embedded rotational discontinuities in nonuniform plasmas and the resultant proton heating, J. Geophys. Res., 106, 5661.

Verkhoglyadova, O., B. Dasgupta, and B. T. Tsurutani (2003), Model for vortex turbulence with discontinuities in the solar wind, Nonlinear Proc. Geophys., 10, 335.

Wilcox, J. M., and P. H. Scherrer (1972), An annual and solar magnetic cycle variations in the interplanetary magnetic field, 1926-1971, J. Geophys. Res., 77, 5385

Winterhalter, D., E. J. Smith, M. E. Burton, N. Murphy, and D. J. McComas (1994a), The heliospheric plasma sheet, J. Geophys. Res., 99, 6667. 
Winterhalter, D., M. Neugebauer, B. E. Goldstein, E. J. Smith, S. J. Bame, and A. Balogh (1994b), Ulysses field and plasma observations of magnetic holes in the solar wind and their relation to mirror-mode structures, J. Geophys. Res., 99, 23,372.

Wrenn, G. L. (1995), Conclusive evidence for internal dielectric charging anomalies on geosynchronous communications spacecraft, J. Spacecr. Rockets, 32, 514.

A. L. C. Gonzalez, W. D. Gonzalez, and F. L. Guarnieri, Instituto Nacional Pesquisas Espaciais (INPE), Caixa Postal 515,1220-970 Sao Jose dos Campos, Sao Paolo, Brazil.

N. Gopalswamy, NASA Goddard Space Flight Center, Code 695.0, Building 21, Room 260, Greenbelt, MD 20771, USA.

M. Grande, Planets and Magnetospheres Group, Rutherford Appleton Laboratory, Chilton, Didcot, Oxfordshire, OX11 0QX, UK.
Y. Kamide, Solar-Terrestrial Environment Laboratory, Nagoya University, Honohara 3-13, Toyokawa, Aichi 442-8507, Japan.

Y. Kasahara, Department of Information and Systems Engineering, Kanazawa University, Kakuma, Kanazawa 920-1192, Japan.

G. Lu, High Altitude Observatory, National Center for Atmospheric Research, 3080 Center Green, Boulder, CO 80301, USA.

I. Mann, Department of Physics, University of Calgary, Edmonton, Alberta, Canada T6G 2J1

R. McPherron, Institute of Geophysics and Planetary Physics, University of California, Los Angeles, 405 Hilgard Avenue, Los Angeles, CA 90024, USA.

F. Soraas, Department of Physics and Technology, University of Bergen, Allegaten 55, N-5007 Bergen, Norway.

B. T. Tsurutani, Jet Propulsion Laboratory, MS 169-506,4800 Oak Grove Drive, Pasadena, CA 91109, USA. (bruce.tsurutani@jpl.nasa.gov)

V. Vasyliunas, Max Planck Institute for Solar System Research, D-37195 Katlenburg-Lindau, Germany. 